\title{
2007/77
}

A fair solution to the compensation problem

\author{
Giacomo Valletta
}




\title{
CORE DISCUSSION PAPER
}

2007/77

\section{A fair solution to the compensation problem}

\author{
Giacomo VALLETTA ${ }^{1}$
}

September 2007

\begin{abstract}
We study equity in economies where agents are endowed with different, non transferable, personal talents. To compensate them for such differences a given amount of money needs to be shared among them. We axiomatize a family of social orderings over allocations based on efficiency, fairness and robustness properties. Taking into account incentive constraints we derive the optimal policy: individuals with the same talent need to be equally compensated and only people whose level of talent is below a certain threshold should receive a positive compensation.
\end{abstract}

Keywords: compensation, talents, fairness, axioms, social orderings, incentivecompatibility.

JEL Classification: D63, D71

${ }^{1}$ CORE, Université catholique de Louvain, Belgium. E-mail: giacomo.valletta@uclouvain.be.

I wish to thank François Maniquet for many helpful discussions and detailed suggestions. I also thank Efthymios Athanasiou, Marc Fleurbaey, Juan D. Moreno-Ternero and the participants to workshops and conferences in Maastricht, Istanbul, Pavia, Louvain-la-Neuve, Hamburg and Marseille for their comments.

This paper presents research results of the Belgian Program on Interuniversity Poles of Attraction initiated by the Belgian State, Prime Minister's Office, Science Policy Programming. The scientific responsibility is assumed by the author. 
We study the fair allocation of a given amount of a one-dimensional, divisible resource (e.g. money) among a finite population of individuals in order to compensate them for their differences in a non-transferable, personal endowment. Such a personal endowment can be either an innate talent or a handicap and can be exemplified by features of human capital like health condition, bodily characteristics or social background.

For instance, one could think of the problem of a social planner willing to provide assistance to people with disabilities by distributing among them a given amount of available resources.

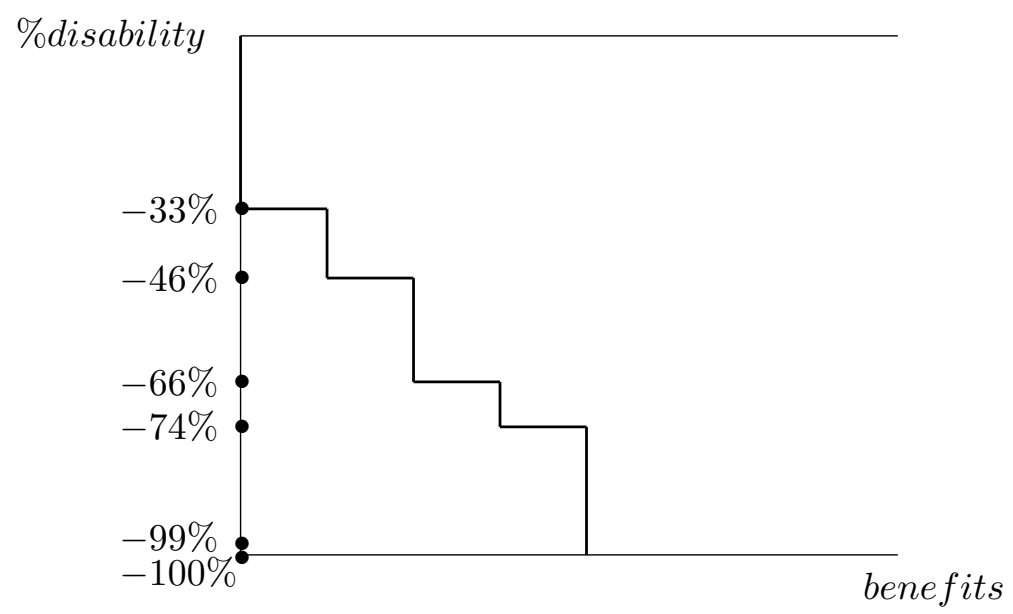

Figure 1: The Italian social security scheme of benefits for people with disabilities.

In several countries, doctors evaluate the mental and physical condition of individuals with handicaps. An index is thus computed which determines to which extent the applicant is able to work. Some benefits are then assigned as a function of this parameter. For example, the compensation scheme used in Italy is illustrated in figure $1^{1}$. The vertical axis represents the degree of inability to work, whereas the horizontal axis represents bundles of benefits. The shape of the curve gives us a clear idea of how the compensation policy is designed: the bundle of benefits grows cumulatively as the level of handicap increases. A significant part of the applicants, people with a level of disability between 0 and $32 \%$, do not receive any kind

\footnotetext{
${ }^{1}$ The website http://www.disabili.it gives a precise description of the compensation scheme, the figure is mine and it has only a descriptive purpose.
} 
of compensation. People with a level of disability between $33 \%$ and $73 \%$ are entitled to some monetary and non-monetary benefits that cumulate depending to the severity of their condition (i.e., specific medical supplies, priority in the unemployment, free drugs). People with a disability between $74 \%$ and $99 \%$ also receive a monthly monetary subsidy but only until their retirement age and provided their earnings are below a certain threshold. Individuals with a disability of $100 \%$ receive the same amount of money permanently and independetly of their wealth condition. The figure raises several questions. Is it fair to leave a relatively big share of the population without any compensation? Is the compensation individuals with severe disabilities receive enough?

We will show that normative arguments and incentive-compatibility constraints give a partial justification to such a compensation scheme. In particular, it might indeed be optimal to give a monetary compensation only to people whose level of disability is above a certain threshold. Such a threshold depends not only on the relative scarcity of the resources to share, but also on the willingness of the social planner to give priority to individuals with higher disability. On the other hand the strong discontinuities in such a scheme, namely, the fact that two individuals with a non-negligible difference in their level of talent might end up receiving the same compensation, does not find any support in our analysis.

Recent theories of justice agree that inequalities in agents'outcomes might arise both from differences in characteristics they should not be held responsible for and from differences in characteristics they should be held responsible for. They all arrive at the common conclusion that society should only try to reduce inequalities deriving from differences of the former type ${ }^{2}$.

We will assume that each individual is endowed with two characteristics: her talent $t$, for which she is not responsible and her preferences $R$ for which she is, since they embody her subjective evaluation of her condition (namely the amount of resources she receives and her talent).

The purpose of our analysis is to look, on one side, for a distribution mechanism that would allocate a given amount of available external resources $M$ in order to correct inequalities deriving from differences in talent solely. This idea goes under the name of principle of compensation (Fleurbaey [7], [8]). Notice that in performing such a compensation we cannot disregard individual preferences: if all the individuals in a society deem equivalent being endowed with a given level of talent, rather than another

\footnotetext{
${ }^{2}$ See for instance Rawls [20], Dworkin [6], Arneson [1], Cohen [4], van Parijs [24], Roemer [21] among others.
} 
one, then differences in talents do not need to be compensated.

On the other side, we endorse the idea that differences due to characteristics that fall into the domain of responsibility should be treated neutrally: this means that, in our case, the distribution mechanism should give the same amount of resources to individuals with the same talent (but possibly different preferences). This is the so called principle of natural reward (Fleurbaey [7], [8])

To solve this problem, the literature has so far proposed allocation rules (for a complete survey of the subject see Fleurbaey and Maniquet [11]). An allocation rule specifies the set of optimal allocations as a function of the parameters of the problem: the population's profile of talents, the population's profile of preferences and the available resources. Such allocation rules can be enforced only on the basis of a full knowledge of the population's profiles of characteristics. Typically allocation rules are not perfectly implementable because of incentive constraints.

Alternatively, one may look for social ordering functions specifying a complete ranking of all the allocations as a function of the parameters of the problem.

This approach has been already applied to several economic environments: for instance Maniquet and Sprumont [17], [18] construct orderings of allocations in economies with one private good and one partially excludable nonrival good. Fleurbaey and Maniquet [13], [14] consider a model where agents have unequal production skills and different preferences and they characterize purely ordinal social ordering functions which satisfy properties of compensation for inequalities in skills and equal access to resources for all preferences. Fleurbaey and Maniquet [12] construct social preferences in the canonical economic problem of dividing a bundle of commodities among individuals with different preferences.

In this paper, we follow the same approach by proposing a way to rank alternatives based on efficiency, fairness and robustness properties. It is explained below how it is possible to embody the principle of compensation and the principle of natural reward into a variety of specific, precise axioms. It will be clear soon that it is not possible to find a social ordering function that satisfies both the principles. Let us immediately emphasize that such an incompatibility does not represent a dead end for our analysis. It is a purely introductory result telling us that we have to make a choice between these two incompatible principles: we have to chose which one should have priority over the other. We will show how axioms embodying the principle of compensation and axioms weakly embodying the principle of natural reward together with efficiency and separability conditions (with some vari- 
ants, separability conditions state that indifferent agents should not matter in the social evaluation of two alternatives) single out a specific welfare representation of agents' preferences and a specific way of ranking alternatives.

Once an ordering of the allocations is available, it is always possible to produce a precise policy recommendation, even if the social planner faces informational constraints (or any other kind of constraint). We will show in fact, that maximizing the ordering, under the relevant incentive constraints, gives us, as mentioned earlier, a specific suggestion about the way resources should be allocated.

The paper is organized as follows. Section 1 introduces the model and the relevant notation. Section 2 describes the requirements imposed on the social preferences and describes logical relations between them. Section 3 deals with social orderings. Section 4 studies the optimal allocation of resources under informational constraints. Section 5 concludes. The Appendix provides the proofs.

\section{The Model}

The model we analyze is derived from Fleurbaey [7], [8]. We consider a set of economies with a finite set of agents $N \subseteq \mathbb{N}$. A given amount $M \in$ $\mathbb{R}_{++}$has to be shared among them through monetary transfers of amount $m_{i} \in \mathbb{R}_{+}$. Each agent in the economy is characterized by a fixed, non transferable talent $t_{i} \in T$. Let $T=\left[t^{L}, t^{H}\right] \subset \mathbb{R}$ be the set of possible talents. Moreover each agent is endowed with a personal preference ordering $R_{i}$ over pairs $(m, t) \in \mathbb{R}_{+} \times T$. A pair $(m, t)$ will be called hereafter an extended bundle. This means that individuals are assumed to be able to compare extended bundles of individuals with a talent different from their own. In this framework one can think of preferences as to valuation functions: each individual is able to give a valuation of her own condition and compare it with someone else's. The individual preference ordering $R_{i}$ is assumed to be continuous and strictly monotonic with respect to $m_{i}$ and $t_{i}$, strict preference and indifference will be respectively denoted by $P_{i}$ and $I_{i}$. Let $\mathcal{R}$ denote the set of such preferences. We will say that individual preferences $R \in \mathcal{R}$ exhibit a higher aversion to lack of talent than $R^{\prime} \in \mathcal{R}\left(R \succ_{A} R^{\prime}\right)$ if they satisfy the following property: for any $t, t^{\prime} \in T$ and for any $m, m^{\prime} \in \mathbb{R}_{+}^{N}$ we have

$$
\begin{aligned}
t^{\prime} & >t \text { and }\left(m^{\prime}, t^{\prime}\right) R^{\prime}(m, t) \\
t^{\prime}<t \text { and }\left(m^{\prime}, t^{\prime}\right) R(m, t) & \Longrightarrow\left(m^{\prime}, t^{\prime}\right) P(m, t) \\
& \left(m^{\prime}, t^{\prime}\right) P^{\prime}(m, t)
\end{aligned}
$$


that is, any pair of indifference curves, one for $R$ and one for $R^{\prime}$ cross at most once in the $(m, t)$ space.

An economy is denoted by $e=\left(t_{N}, R_{N}, M\right)$, where $t_{N}=\left(t_{i}\right)_{i \in N}$ is the population's profile of talents, $R_{N}=\left(R_{i}\right)_{i \in N}$ is the profile of individual preferences and $M$ is the available social endowment. The domain of all economies satisfying the above assumptions will be denoted by $\mathcal{D}$. An allocation is a vector $m_{N}=\left(m_{i}\right)_{i \in N} \in \mathbb{R}_{+}^{N}$. The first concern of this paper is to construct a social ordering of (feasible and non feasible) allocations for all economies in the domain. A social ordering, for any given $e \in \mathcal{D}$, is a complete and transitive ranking defined over allocations. A social ordering function $\bar{R}$ associates every admissible economy $e=\left(t_{N}, R_{N}, M\right) \in \mathcal{D}$ with a social ordering $\bar{R}(e)$. So for an economy $e=\left(t_{N}, R_{N}, M\right) \in \mathcal{D}$ and two allocations $m_{N}$ and $m_{N}^{\prime} \in \mathbb{R}_{+}^{N}$ we write $m_{N} \bar{R}(e) m_{N}^{\prime}$ to denote that $m_{N}$ is (socially) at least as good as $m_{N}^{\prime}$. Strict social preference and indifference will be respectively denoted by $\bar{P}(e)$ and $\bar{I}(e)$.

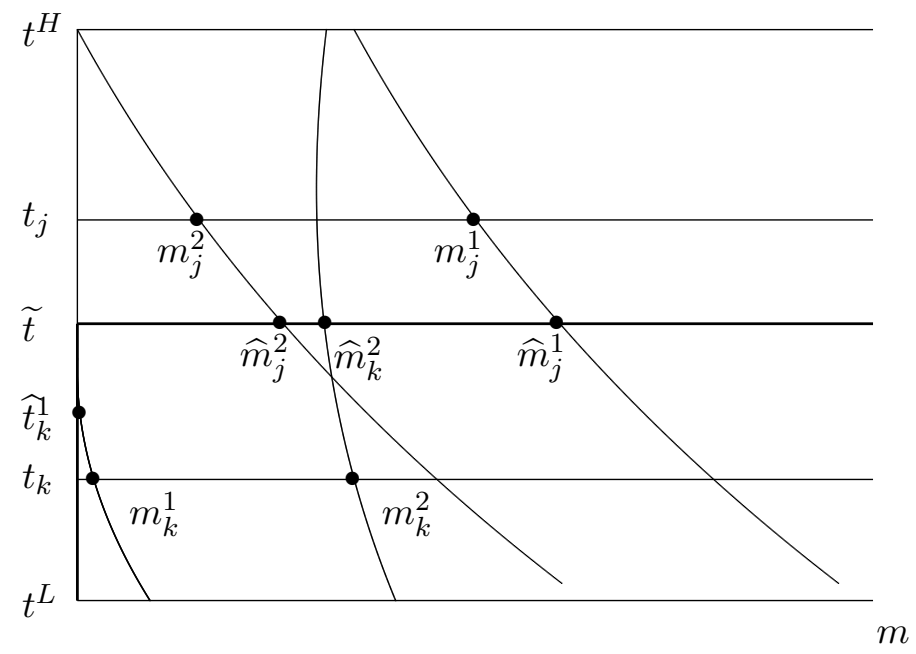

Figure 2:

To illustrate our approach consider the simple economy depicted in figure 2. We only have two agents, $j$ and $k$, with different talents, $t_{j}$ and $t_{k}$. We consider two allocations, $\left(m_{j}^{1}, m_{k}^{1}\right)$ and $\left(m_{j}^{2}, m_{k}^{2}\right)$ and we want to rank them. The social ordering function we propose in this paper works in the following way. First, a reference talent $\widetilde{t}$ is chosen. Then, for each $i \in\{j, k\}, q \in\{1,2\}$ we consider the individual level of resource $\widehat{m}_{i}^{q} \geq 0$ which would make the agents accept the reference talent $\widetilde{t}$ instead of their current situation $\left(m_{i}^{q}, t_{i}\right)$. As we can see from the figure, such a value does not exist for agent $k$ at 
$\left(m_{j}^{1}, m_{k}^{1}\right)$. In order to evaluate the situation of agent $k$, we then consider the level of talent $\widehat{t}_{k}^{1}$ that would make her accept a null transfer instead of her current situation. More formally, $\widehat{t}_{k}^{1}$ is the level of talent such that $\left(0, \widehat{t}_{k}^{1}\right) I_{k}\left(m_{k}^{1}, t_{k}\right)$. To summarize, for each agent $i$ we can have a continuous numerical representation of her preferences over pairs $(m, t) \in \mathbb{R}_{+} \times T$ :

$$
\widehat{E}_{R_{i}, \widetilde{t}}\left(m_{i}, t_{i}\right)= \begin{cases}\widehat{m}_{i} & \text { if } \widehat{m}_{i} \text { exists } \\ \widehat{t}_{i}-\widetilde{t} & \text { otherwise. }\end{cases}
$$

Finally the vectors of such welfare representations are compared using the maximin rule: in our example we have that $\left(m_{j}^{2}, m_{k}^{2}\right)$ is strictly better than $\left(m_{j}^{1}, m_{k}^{1}\right)$ since $\min \left\{\widehat{E}_{j}^{2}, \widehat{E}_{k}^{2}\right\}>\min \left\{\widehat{E}_{j}^{1}, \widehat{E}_{k}^{1}\right\}$. We will prove in Section 4 that both this particular welfare representation and the way of ranking alternatives are singled out by a specific combination of axioms. More precisely this is what we obtain if we combine axioms embodying the principle of compensation, axioms weakly embodying the principle of natural reward together with efficiency and robustness requirements. Indeed, if we measured welfare differently, keeping the same ranking criterion, we would not be able to obtain a compensation minded social ordering function. Consider, for example, the following way of ranking alternatives. Take two allocations $m_{N}$ and $m_{N}^{\prime}$ and order the extended bundles according to a reference preference $\widetilde{R} \in \mathcal{R}$ in such a way that $\left(m_{i}, t_{i}\right) \widetilde{R}\left(m_{i+1}, t_{i+1}\right)$ and $\left(m_{i}^{\prime}, t_{i}\right) \widetilde{R}\left(m_{i+1}^{\prime}, t_{i+1}\right)$ for all $i \in(1, \ldots, n-1)$ then, $m_{N}^{\prime}$ is socially (strictly) preferred to $m_{N}$ if $\left(m_{n}^{\prime}, t_{n}\right) \widetilde{P}\left(m_{n}, t_{n}\right)^{3}$. This social ordering function fails to satisfy the principle of compensation (except for agents whose individual preference ordering is equal to the reference one) and on the contrary gives priority to the principle of natural reward.

This duality is actually due to a basic incompatibility between the two ethical principles that was already pointed out by Fleurbaey [8] in the first best framework and, as will be proven in section 3 , it is still present in the second best framework.

On the other hand, suppose we keep the welfare representation proposed in the first place but we drop the maximin criterion. We could, for example, take the welfare-level vectors underlying two different allocations and calculate their Gini index (we will consider only the striclty positive elements). The allocation with the smallest Gini index will be socially preferred to the other one. This ranking criterion is, to some extent, compensation minded

\footnotetext{
${ }^{3}$ notice that the agents with the worst-off extended bundles respect to $\widetilde{R}$, at the two allocations, are not necessarily the same.
} 
but it is not necessarily consistent with an infinite degree of inequality aversion. As we will see later this is a feature we are forced to accept if we combine together compensation and robustness requirements.

\section{Axioms}

This section defines the properties we want to impose on our social ordering function. We start with a basic efficiency condition. The Strong Pareto axiom takes a very intuitive form here since talents are fixed and resources are one-dimensional. Notice moreover that all allocations exhausting the resources are efficient.

Strong Pareto. For all $e \in \mathcal{D}$ and $m_{N}, m_{N}^{\prime} \in \mathbb{R}_{+}^{N}$, if $m_{i} \geq m_{i}^{\prime}$ for all $i \in N$, then $m_{N} \bar{R}(e) m_{N}^{\prime}$; if, in addition, $m_{i}>m_{i}^{\prime}$ for some $i \in N$, then $m_{N} \bar{P}(e) m_{N}^{\prime}$.

The second axiom we introduce is a property of robustness of the social ordering with respect to changes in the set of agents. We require that adding or removing agents who receive the same bundle in two different allocations should not modify the social ordering. This axiom was introduced by Fleurbaey and Maniquet [12] and is reminiscent of the Separability condition, quite familiar in social choice, due to d'Aspremont and Gevers [2] and of the Consistency condition, widely used in the theory of fair allocation (see Thomson [23]), except that it does not require to delete the resources consumed by the removed agents from the social endowment.

Separation. For all $e \in \mathcal{D}$ and $m_{N}, m_{N}^{\prime} \in \mathbb{R}_{+}^{N}$, if there is $i \in N$ such that $m_{i}=m_{i}^{\prime}$, then

$$
m_{N} \bar{R}(e) m_{N}^{\prime} \Longleftrightarrow m_{N \backslash\{i\}} \bar{R}\left(t_{N \backslash\{i\}}, R_{N \backslash\{i\}}, M\right) m_{N \backslash\{i\}}^{\prime} .
$$

We can now turn to the fairness conditions expressing respectively the ideas of compensation and natural reward. Assume first that there are two agents, $j$ and $k$, with equal preferences and a different talent. Assume also that they both consider agent $j$ 's extended bundle strictly better than agent $k$ 's. Assume finally that the monetary compensation received by agent $j$ is reduced by a given amount and the one received by agent $k$ is increased by the same amount but they both still consider $j$ 's extended bundle to be better than $k$ 's. The allocation we obtain must be, according to the principle of compensation, socially preferred to the former one since it reduces 
inequalities exclusively deriving from differences in talent.

Equal Preferences Transfer. For all $e \in \mathcal{D}$ and $m_{N}, m_{N}^{\prime} \in \mathbb{R}_{+}^{N}$, if there exist $j, k \in N$ and $\Delta \in R_{+}$such that $R_{j}=R_{k}$,

$$
m_{j}^{\prime}=m_{j}-\Delta, m_{k}^{\prime}=m_{k}+\Delta \text { and }\left(m_{j}^{\prime}, t_{j}\right) P_{j}\left(m_{k}^{\prime}, t_{k}\right),
$$

with $m_{i}=m_{i}^{\prime}$ for all $i \neq j, k$, then $m_{N}^{\prime} \bar{P}(e) m_{N}$.

We also consider a quite natural strengthening of this axiom: instead of requiring the preferences of the agents to be identical, we simply require that agents' indifference curves trough their extended bundles in $m_{N}$ and $m_{N}^{\prime}$ are nested. For each $i \in N$ let $L\left(\left(m_{i}, t_{i}\right), R_{i}\right)$ and $U\left(\left(m_{i}, t_{i}\right), R_{i}\right)$ denote respectively the (closed) lower contour set and the (closed) upper contour set of $R_{i}$ at $\left(m_{i}, t_{i}\right)$.

Nested Preferences Transfer. For all $e \in \mathcal{D}$ and $m_{N}, m_{N}^{\prime} \in \mathbb{R}_{+}^{N}$ if there exist $j, k \in N$ and $\Delta \in R_{+}$such that

$$
U\left(\left(m_{j}^{\prime}, t_{j}\right), R_{j}\right) \cap L\left(\left(m_{k}^{\prime}, t_{k}\right), R_{k}\right)=\emptyset,
$$

$m_{j}^{\prime}=m_{j}-\Delta$ and $m_{k}^{\prime}=m_{k}+\Delta$, with $m_{i}=m_{i}^{\prime}$ for all $i \neq j, k$, then $m_{N}^{\prime} \bar{P}(e) m_{N}$.

The rationale of this axiom is that, in both allocations, one of the two agents envies the other (not only at her actual talent but also at any hypothetical level of talent she may have), so performing the transfer actually reduces resources inequality.

Let us turn now to the principle of natural reward. Assume there are two agents with the same talent (and possibly different preferences) who receive a different monetary compensation: performing an equalizing transfer that reduces such an inequality should be considered a social improvement.

Equal Talent Transfer. For all $e \in \mathcal{D}$ and $m_{N}, m_{N}^{\prime} \in \mathbb{R}_{+}^{N}$, if there exist $j, k \in N$ and $\Delta \in R_{+}$such that $t_{j}=t_{k}$ and

$$
m_{k}+\Delta=m_{k}^{\prime}<m_{j}^{\prime}=m_{j}-\Delta,
$$

with $m_{i}=m_{i}^{\prime}$ for all $i \neq j, k$, then $m_{N}^{\prime} \bar{P}(e) m_{N}$.

The fairness conditions presented so far are an adaptation to this framework of the Pigou-Dalton principle of transfer, widely used in income inequality 
theory. They are all biased toward equality but consistent with any degree of inequality aversion.

Our first result shows that the compensation and natural reward requirements we have presented so far are incompatible.

Lemma 1 On the domain $\mathcal{D}$, no social ordering function satisfies Equal Preferences Transfer and Equal Talent Transfer.

We propose now an alternative way of capturing the ethical goals of compensation and natural reward that is inspired by Suppes' grading principles (Suppes [22]) and was introduced in the literature by Maniquet [16]. The first property, called Equal Preferences Permutation, requires that permuting the outcomes of two agents having the same preferences but possibly different talents gives us two socially equivalent allocations. Indeed, according to the principle of compensation there is no reason why society should prefer one of the two allocations: agents with the same responsibility characteristics should be treated anonymously.

Equal Preferences Permutation. For all $e \in \mathcal{D}$ and $m_{N}, m_{N}^{\prime} \in \mathbb{R}_{+}^{N}$, if there exist $j, k \in N$ such that $R_{j}=R_{k}$,

$$
\left(m_{j}, t_{j}\right) I_{j}\left(m_{k}^{\prime}, t_{k}\right) \text { and }\left(m_{k}, t_{k}\right) I_{j}\left(m_{j}^{\prime}, t_{j}\right),
$$

with $m_{i}=m_{i}^{\prime}$ for all $i \neq j, k$, then $m_{N} \bar{I}(e) m_{N}^{\prime}$.

The next axiom requires, according to the principle of natural reward, that permuting the bundles of two agents having the same talent but possibly different preferences does not alter the value of the allocation in the social ranking. Again the justification is clear: as those agents have the same compensation parameters, they should be treated anonymously. By permuting the transfers they receive, we obtain an equally good, or equally bad, allocation.

Equal Talent Permutation. For all $e \in D$ and $m_{N}, m_{N}^{\prime} \in \mathbb{R}_{+}^{N}$, if there exist $j, k \in N$ such that $t_{j}=t_{k}$,

$$
m_{k}=m_{j}^{\prime} \text { and } m_{j}=m_{k}^{\prime},
$$

with $m_{i}=m_{i}^{\prime}$ for all $i \neq j, k$, then $m_{N} \bar{I}(e) m_{N}^{\prime}$.

The next result shows that the incompatibility between compensation and responsibility extends beyond Lemma 1 since it has nothing to do with the 
degree of inequality aversion exhibited by the axioms that were involved there: also requirements like Equal Preferences Permutation and Equal Talent Permutation, which are consistent with any degree of inequality aversion (even a negative one), are in fact incompatible if combined with Strong Pareto.

Lemma 2 On the domain $\mathcal{D}$, no social ordering function satisfies Strong Pareto, Equal Preferences Permutation and Equal Talent Permutation.

Similar incompatibilities between compensation and natural reward, in a second best framework, have already been pointed out by Fleurbaey and Maniquet [13] in a different economic environment.

Let us stress again that these negative results are purely introductory ones, they imply that we have to chose which of the two principles must have priority. As we shall see, this choice has an important consequence concerning the the degree of inequality aversion that will be exhibited finally by the social ordering function. In fact, if we decide to give priority to the principle of compensation, then, combining together a mild egalitarian requirement like Nested Preferences Transfer, with axioms that are essentially neutral with respect to inequality aversion, determines an extreme form of inequality aversion described by the following axiom.

Nested Preferences Priority. For all $e \in \mathcal{D}$ and $m_{N}, m_{N}^{\prime} \in \mathbb{R}_{+}^{N}$, if there exist $j, k \in N$ such that

$$
\begin{aligned}
& \left(m_{j}, t_{j}\right) P_{j}\left(m_{j}^{\prime}, t_{j}\right) P_{j}\left(m_{k}^{\prime}, t_{k}\right) P_{k}\left(m_{k}, t_{k}\right) \\
& U\left(\left(m_{j}^{\prime}, t_{j}\right), R_{j}\right) \bigcap L\left(\left(m_{k}^{\prime}, t_{k}\right), R_{k}\right)=\emptyset,
\end{aligned}
$$

with $m_{i}=m_{i}^{\prime}$ for all $i \neq j, k$, then $m_{N}^{\prime} \bar{P}(e) m_{N}$.

Let us emphasize that this property represents an extreme strengthening of Nested Preferences Transfer. It states that an inequality reduction is always socially desirable, provided that indifference curves are nested at the bundles, even when the gain from $m_{k}$ to $m_{k}^{\prime}$ is very small and the loss from $m_{j}$ to $m_{j}^{\prime}$ is quite large.

Lemma 3 On the domain $\mathcal{D}$, if a SOF satisfies Nested Preferences Transfer, Equal Preferences Permutation and Separation then it satisfies Nested Preferences Priority.

Results similar to this one can already be found in Fleurbay [9] and Maniquet and Sprumont [17], [18]. They all show, in different economic 
environments, that combining together fairness conditions that exhibit a limited aversion to inequality with robustness and efficiency conditions leads to an infinite aversion to inequality.

The remarkable aspect here is that we reach a similar conclusion dealing with a one-dimensional resource ${ }^{4}$. This makes the contrast with the income inequality measurement framework, where combining the PigouDalton transfer principle with robustness requirements does not necessarily lead to such extreme consequences (Chakravarty [5]), even stronger.

It is moreover interesting to notice that if we decide to give priority to the principle of natural reward, we cannot find anything similar. In this case, in fact, we would "fall" in a purely one-dimensional framework were we are eventually free to choose the degree of inequality aversion of the social ordering function ${ }^{5}$.

The fact that we have decided to give priority to the principle of compensation does not mean that we want to disregard completely the principle of natural reward. In the last part of this section we will propose two alternative weakenings of Equal Talent Transfer that represent somehow the strongest natural reward axioms that would not clash with Equal Preferences Transfer.

The impossibility pointed out by Lemma 1 makes it clear that, if we do not want to violate Equal Preferences Transfer, then it is possible to perform a redistribution between two agents with the same talent $t$ who receive a different monetary compensation at most for one $t \in T$. A first option then, is to impose the full transfer condition only among agents with a talent equal to some reference talent $\widetilde{t}$ fixed arbitrarily. Alternatively, if there are two agents $j, k$ such that $t_{j}=t_{k}<\tilde{t}$ and one of them does not receive a monetary compensation, while the other receives a positive one, then we propose a minimal reward requirement: it must be always possible to slightly reduce such a difference and the allocation we obtain is socially preferred to the former one.

\footnotetext{
${ }^{4}$ Interestingly a similar result involving Equal Preference Transfer (and its Priority version) could be obtained if we replace Separation with a different informational simplicity axiom: Hansson Independence (Fleurbaey and Maniquet [14]). It says that the social ranking of two allocations should remain unaffected by a change in individual preferences which does not modify agents' indifference curves at the bundles they receive in these two allocations. Indeed if a social ordering function satisfies Equal Preferences Transfer, Equal Preferences Permutation and Hannson Independence then it also satisfies Equal Preferences Priority.

${ }^{5}$ We could, for example, compare allocations just by computing their Gini index. This would satisfy Strong Pareto and Equal Talent Transfer (but it would violate Equal Preferences Transfer).
} 
$\tilde{t}$-Equal Talent Transfer. For all $e \in \mathcal{D}, m_{N}, m_{N}^{\prime} \in \mathbb{R}_{+}^{N}$, for at least one $\widetilde{t} \in T$ if there exist $j, k \in N$ and $\Delta \in R_{+}$such that $t_{j}=t_{k}=\widetilde{t}$ and $m_{j}-\Delta=m_{j}^{\prime}>m_{k}^{\prime}=m_{k}+\Delta$, with $m_{i}=m_{i}^{\prime}$ for all $i \neq j, k$, then $m_{N}^{\prime} \bar{P}(e) m_{N}$

or

if there exist $j, k \in N$ such that $t_{j}=t_{k}<\tilde{t}, m_{j}=0, m_{k}>0$ with $m_{i}=m_{i}^{\prime}$ for all $i \neq j, k$, then there exists at least one $\varepsilon \in \mathbb{R}_{++}$such that $\varepsilon=m_{j}^{\prime}<m_{k}^{\prime}=m_{k}-\varepsilon$ and $m_{N}^{\prime} \bar{P}(e) m_{N}$.

The next axiom does not involve the choice of a reference talent. We still consider a couple of agents with the same talent who receive a different amount of resources. We say that it is socially desirable to perform a transfer between them only if the recipient's preferences exhibits a higher degree of aversion to lack of talent than the donor's preferences. Alternatively, if there are two agents $j, k$ such that $t_{j}=t_{k}$ and one of them does not receive a monetary compensation, while the other receives a positive one, then it must be always possible to slightly reduce such a difference while obtaining a social improvement.

Equal Talent Transfer to the Unhappy. for all $e \in \mathcal{D}$ and $m_{N}, m_{N}^{\prime} \in$ $\mathbb{R}_{+}^{N}$, if there exist $j, k \in N$ and $\Delta \in R_{+}$such that $t_{i}=t_{j}, R_{j} \succ_{A} R_{i}$ and $m_{k}+\Delta=m_{k}^{\prime}<m_{j}^{\prime}=m_{j}-\Delta$, with $m_{i}=m_{i}^{\prime}$ for all $i \neq j, k$, then $m_{N}^{\prime} \bar{P}(e) m_{N}$

or

if there exist $j, k \in N$ such that $t_{j}=t_{k}, m_{j}=0, m_{k}>0$ with $m_{i}=m_{i}^{\prime}$ for all $i \neq j, k$, then there exists at least one $\varepsilon \in \mathbb{R}_{++}$such that: $\varepsilon=m_{j}^{\prime}<$ $m_{k}^{\prime}=m_{k}-\varepsilon$ and $m_{N}^{\prime} \bar{P}(e) m_{N}$.

\section{Social Orderings}

Before we show how combining together the axioms presented in the previous section determines a specific way of ranking the social alternatives, we need 
to introduce some piece of notation.

Assume a reference talent $\widetilde{t}$ is chosen. For each agent $i \in N$ and for each $m_{i} \in \mathbb{R}_{+}$consider $\widehat{m}_{i} \geq 0$ such that $\left(m_{i}, t_{i}\right) I_{i}\left(\widehat{m}_{i}, \widetilde{t}\right)$. As pointed in the example in section 2 , such a value does not necessarily exist. If this is the case, consider the hypothetical level of talent $\widehat{t}_{i}$ such that $(0, \widehat{t}) I_{i}\left(m_{i}, t_{i}\right)$. Finally, for each $i \in N$ consider the function

$$
\widehat{E}\left(m_{i}, t_{i}, R_{i}, \widetilde{t}\right)= \begin{cases}\widehat{m}_{i} & \text { if } \widehat{m}_{i} \text { exists } \\ \widehat{t}_{i}-\widetilde{t} & \text { otherwise }\end{cases}
$$

It is worth to stress again that such a value is a continuous numerical representation of the preferences of the agents over extended bundles. Hence for each allocation we will have a vector of such welfare levels. It turns out that if we pursue the objective of giving priority to the principle of compensation then such vectors need to be compared using the maxmin rule.

Theorem 1 Let a Social Ordering Function satisfy Strong Pareto, Nested Preferences Transfer, Equal Preferences Permutation, $\widetilde{t}$-Equal Talent Transfer and Separation. Then, on the domain $\mathcal{D}$, for any $m_{N}, m_{N}^{\prime} \in \mathbb{R}_{+}^{N}$, one has $m_{N}^{\prime} P(e) m_{N}$ if

$$
\min _{i} \widehat{E}\left(m_{i}^{\prime}, t_{i}, R_{i}, \widetilde{t}\right)>\min _{i} \widehat{E}\left(m_{i}, t_{i}, R_{i}, \widetilde{t}\right)
$$

The main drawback of Theorem 1 is that the evaluation function of each agent depends on the value of the reference talent $\widetilde{t}$ that is exogenously fixed by the social planner. So, a different choice of $\widetilde{t}$ might lead to a different social ordering. We can overcome this difficulty by substituting $\widetilde{t}$-Equal Talent Transfer with Equal Talent Transfer to the Unhappy. Using this particular weakening of Equal Talent Transfer we are able to endogenize the choice of the reference talent. More precisely, the introduction of Equal Talent Transfer to The Unhappy leads to select $t^{H}$ as the reference talent.

Theorem 2 Let a Social Ordering Function satisfy Strong Pareto, Nested Preferences Transfer, Equal Preferences Permutation, Equal Talent Transfer to the Unhappy and Separation. Then, on the domain $\mathcal{D}$, for any $m_{N}, m_{N}^{\prime} \in \mathbb{R}$, one has $m_{N}^{\prime} P(e) m_{N}$ if

$$
\min _{i} \widehat{E}\left(m_{i}^{\prime}, t_{i}, R_{i}, t^{H}\right)>\min _{i} \widehat{E}\left(m_{i}, t_{i}, R_{i}, t^{H}\right)
$$


The fact that, both for Theorem 1 and Theorem 2, no axiom is redundant, will be shown in the appendix.

Similar results can be found in Fleurbaey and Maniquet [14] and Maniquet and Sprumont [17], [18]. They propose maximin criteria that like ours rely only on ordinal, non-interpersonally comparable, individual preferences.

No ex-ante information about individual utilities is needed in order to build the social ranking we propose: on the contrary, our axioms single out a particular welfare representation of individual preferences. The corresponding welfare-level vectors need then to be ranked according to the maximin criterion: a way of ranking alternatives that gives priority to agents with a low $\widehat{E}($.$) , that is, either agents with a low m_{i}$ or agents who dislike their level of talent.

None of the theorems provides a full characterization of social preferences because they do not tell us how to rank two allocations for which $\min _{i} \widehat{E}\left(m_{i}^{\prime}, t_{i}, R_{i}, \widetilde{t}\right)=\min _{i} \widehat{E}\left(m_{i}, t_{i}, R_{i}, \widetilde{t}\right)$. Nonetheless, as we will see in the next section, they both give us a criterion to evaluate different compensation policies and, in particular, to single out the optimal one. Moreover, in both cases, the refinement of the proposed social ordering obtained by applying the leximin criterion ${ }^{6}$ to the welfare-level vectors gives us a complete ranking of the social alternatives and, as can be easily checked, satisfies all the involved axioms.

\section{Incentive Compatible Allocations}

With a social ordering function like the one we have introduced in the previous section, we would obtain, at the optimum, an allocation such that each agent is indifferent between the extended bundle she receives and a hypothetical extended bundle which is the same for everyone (assuming that everyone receives a strictly positive transfer). Such an allocation would be achievable if the social planner knew the talent and the preferences of each agent. In our framework we can reasonably assume that the former is known (doctors can establish the degree of disability of an individual) while individual preferences are typically not observable ${ }^{7}$.

\footnotetext{
${ }^{6}$ Let $\geq_{L}$ denote the usual leximin ordering of $\mathbb{R}_{+}^{n}$ : for any $w, w^{\prime} \in \mathbb{R}_{+}^{n}, w^{\prime} \geq_{L} w$ if and only if the smallest coordinate of $w^{\prime}$ is greater than the smallest coordinate of $w$ or they are equal but the second smallest coordinate of $w^{\prime}$ is greater than the second smallest coordinate of $w$ and so on

${ }^{7}$ If the talent was not observable either, the only incentive-compatible allocation would trivially be the one that gives an equal split of the available resources to each agent.
} 


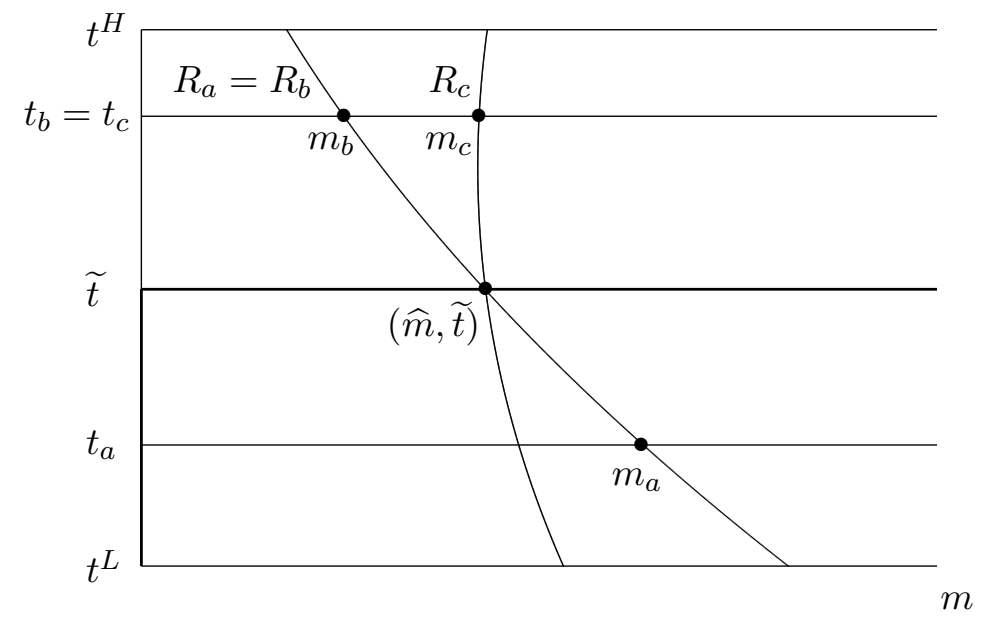

Figure 3: Agent b has an incentive to misreport her preferences.

Consider, for instance, the simple three-agent economy depicted in figure 3. At the optimum, we have full compensation between agent $a$ and agent $b$. On the other hand, agent $b$ and agent $c$, who have different preferences but equal talent, receive a different transfer: $m_{c}>m_{b}$. This clearly gives an incentive to agent $b$ to misreveal her preferences. We can however still use the ranking criterion we have proposed in order to choose the second-best policy.

Interestingly, we will see that the incentive compatibility constraint considerably shapes our choice between the principle of compensation and the principle of natural reward. Actually, from the previous example it is easy to understand that, if the social planner knows the distribution of individual preferences, an allocation $m_{N} \in \mathbb{R}^{N}$ is incentive-compatible if and only if

$$
m_{i}=m_{j} \text { for all } i, j \in N \text { with } t_{i}=t_{j}
$$

that is, agents with the same talent should receive the same amount of resources regardless of their preferences and this is indeed what we should do if we want to give priority to the principle of natural reward.

For any given economy $e \in \mathcal{D}$, let $T^{e}$ and $\mathcal{R}^{e}$ denote, respectively, the set of the different levels of talents and the set of individual preference orderings we can find in $e$. Before we show the shape of the optimal incentive compatible allocation we need to introduce two more assumptions. The first one is nothing but the adaptation to this framework of the Mireless-Spence single crossing condition: for any couple of preferences ordering $R$ and $R^{\prime}$ in $\mathcal{R}^{e}$ we either have that $R$ exhibits a higher degree of inequality aversion to lack 
of talent than $R^{\prime}$ or vice versa. This means that it is possible, within each economy, to rank all the preference orderings with respect to the aversion to lack of talent they exhibit.

Assumption 1: For each $R, R^{\prime} \in \mathcal{R}^{e}$, either $R \succ_{A} R^{\prime}$ or $R^{\prime} \succ_{A} R$.

Let $R^{H}$ and $R^{L}$ denote the preference orderings in $\mathcal{R}^{e}$ with, respectively, highest and lowest aversion to lack of talent.

The second assumption we introduce is a richness requirement that looks quite reasonable specially if the number of agent in the economy is considerably large: for any class of agents with a given talent $t \in T^{e}$ there is at least one agent with preferences $R$, for each $R \in \mathcal{R}^{e}$.

Assumption 2: For each $(t, R) \in T^{e} \times \mathcal{R}^{e}$ there exists $i \in N$ such that $R_{i}=R$ and $t_{i}=t$

Given any reference talent $\widetilde{t}$ chosen from $T$ let $N_{\widetilde{t}}^{+}=\left\{i \in N \mid t_{i} \geq \widetilde{t}\right\}$ and $N_{\widetilde{t}}^{-}=\left\{i \in N \mid t_{i}<\widetilde{t}\right\}$. Consider an incentive-compatible allocation. By Assumption 2 , for each $t \geq \widetilde{t}$, there is at least one agent with such a talent and with preferences equal to $R^{L}$. It is easy to check that, among agents with the same talent, she is the one with lowest $\widehat{E}($.$) . Symmetrically, for each$ possible level of talent $t$, the agents with lowest $\widehat{E}($.$) are those with pref-$ erences $R^{H}$. Since we are using the maximin criterion, we should equalize, whenever possible, the lowest $\widehat{E}($.$) values across different classes.$

Theorem 3 In any economy $e=\left(t_{N}, R_{N}, M\right) \in \mathcal{D}$, under Assumptions 1 and 2, if a Social Ordering Function satisfies Strong Pareto, Nested Preferences Transfer, Equal Preferences Permutation, $\widetilde{t}$-Equal Talent Transfer and Separation then, the optimal incentive compatible allocation $\left(m_{N}^{\widetilde{t}}\right)$ distributes the available resources in the following way:

$$
\text { for all } i, j \in N_{\widetilde{t}}^{+},\left(m_{i}^{\widetilde{t}}, t_{i}\right) I^{L}\left(m_{j}^{\widetilde{t}}, t_{j}\right) \text { or } m_{i}^{\widetilde{t}}=0 \text { and }\left(0, t_{i}\right) R^{L}\left(m_{j}^{\widetilde{t}}, t_{j}\right) .
$$

and

$$
\text { for all } i, j \in N_{\widetilde{t}}^{-},\left(m_{i}^{\widetilde{t}}, t_{i}\right) I^{H}\left(m_{j}^{\widetilde{t}}, t_{j}\right) \text { or } m_{i}^{\widetilde{t}}=0 \text { and }\left(0, t_{i}\right) R^{H}\left(m_{j}^{\widetilde{t}}, t_{j}\right)
$$

Proof. By Theorem 1 we know that if a Social Ordering Function satisfies the listed axioms then, for any $m_{N}^{\prime}, m_{N} \in \mathbb{R}_{+}^{N}$,

$$
\min _{i} \widehat{E}\left(m_{i}^{\prime}, t_{i}, R_{i}, \widetilde{t}\right)>\min _{i} \widehat{E}\left(m_{i}, t_{i}, R_{i}, \widetilde{t}\right) \Longrightarrow m_{N}^{\prime} \bar{P}(e) m_{N}
$$




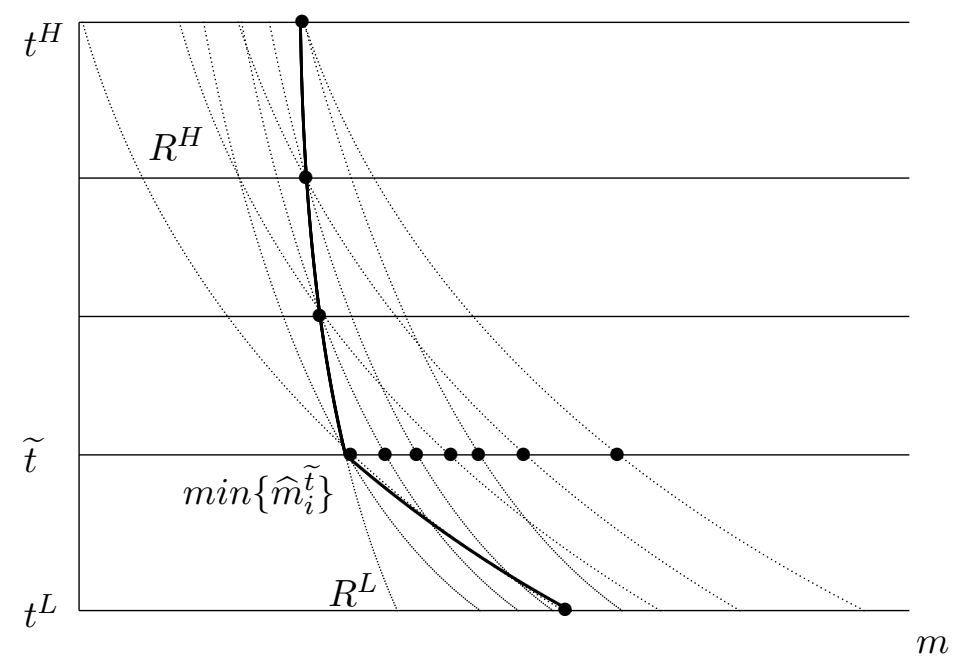

Figure 4: Proof of Theorem 3.

At $m_{N}^{\widetilde{t}}$, given assumption 1 and 2 , it holds true that the worst-off agents are (for a graphical example see figure 4, for simplicity we represent a situation in which each agent receives a strictly positive transfer) all $j \in N_{\widetilde{t}}^{+}$ such that $R_{j}=R^{L}$ and $m_{j}>0$ and all $k \in N_{\tilde{t}}^{-}$such that $R_{k}=R^{H}$ and $m_{k}>0$ :

$$
\min _{i}\left\{\widehat{E}\left(m_{i}^{\widetilde{t}}, t_{i}, \widetilde{t}, R_{i}\right)\right\}_{i \in N}=\widehat{E}\left(m_{j}^{\widetilde{t}}, t_{j}, \widetilde{t}, R^{L}\right)=\widehat{E}\left(m_{k}^{\widetilde{t}}, t_{k}, \widetilde{t}, R^{H}\right) .
$$

Assume, by contradiction, that there exists an incentive compatible allocation $m_{N}^{\prime}$ such that $\min _{i} \widehat{E}\left(m_{i}^{\prime}, t_{i}, R_{i}, \widetilde{t}\right)>\min _{i} \widehat{E}\left(m_{i}^{\widetilde{t}}, t_{i}, R_{i}, \widetilde{t}\right)$. This implies that, for all $j \in N_{\widetilde{t}}^{+}$such that $R_{j}=R^{L}$ and $m_{j}^{\prime}>0, m_{j}^{\prime}>m_{j}^{\widetilde{t}}$ and, analogously, for all $k \in N_{\tilde{t}}^{-}$such that $R_{h}=R^{H}$ and $m_{k}^{\prime}>0, m_{k}^{\prime}>m_{k}^{\widetilde{t}}$. By incentive compatibility we should then increase by the same amount the transfer received by all the other agents having the same level of talent but different preferences. This contradicts that, by construction, $m_{N}^{\widetilde{t}}$ is an allocation exhausting the available resources.

The resulting allocation is strongly affected by the incentive compatibility constraint: people with the same talent receive the same monetary transfer. The scheme we obtain is hence natural-reward minded, nonetheless it shows us the highest level of priority that we can give to the principle of compensation given the informational constraints we have to face. 


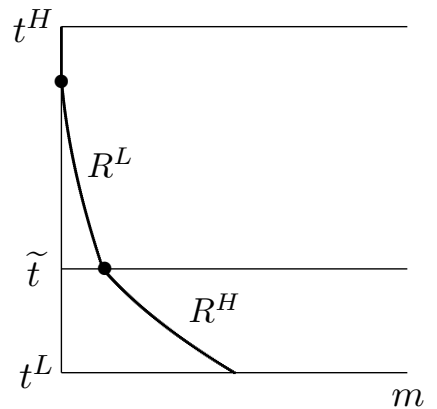

(a)

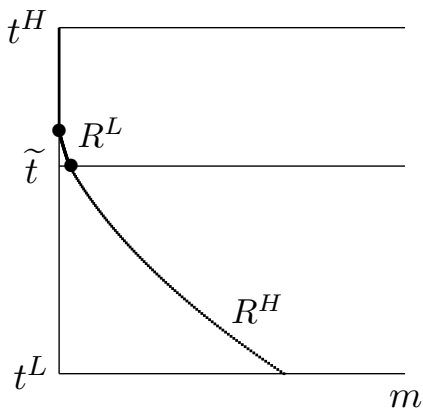

(b)

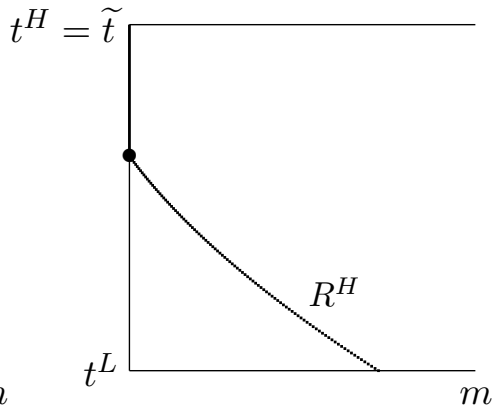

(c)

Figure 5: A different reference talent determines a different compensation policy.

A consequence of Theorem 3 is that the optimal compensation path crucially depends on the choice of $\widetilde{t}$. So, for example, the higher is the reference talent chosen by the social planner the bigger is the compensation received by people with a severe disability (see figure 5 (a) and (b)). Moreover, the proportion of individuals with a low disability is typically higher than the proportion of agents with a severe disability and the equality of extended bundles (with respect to the references preferences $R^{H}$ and $R^{L}$ ) is performed here in such a way that it may be impossible to fully compensate some inequalities in personal characteristics with the available resources. In that case, some inequalities persist and the better-off agents are left with no external resource, while only disadvantaged agents receive a strictly positive monetary compensation. The higher $\widetilde{t}$ the more this is likely to happen: this, in fact, determines a threshold above which $m=0$. It is clear that this gives justification to several compensation schemes used in reality that choose to leave with no transfer people with a relatively high talent. Moreover it is also interesting to note that the compensation scheme is relatively smooth if compared with compensation schemes we can observe in reality: the amount received varies with the level of talent and it is never the case that people with a different $t$ receive the same transfer (except, of course, those who are not compensated at all).

At this point it is quite simple to understand the policy implications of Theorem 2. As we have seen in this case the reference talent is endogenously determined and is equal to $t^{H}$. This means that the compensation policy can no longer be arbitrarily changed by varying the reference talent but fol- 
lows a path that is endogenously determined.

Corollary 1. In any economy $e=\left(t_{N}, R_{N}, M\right) \in \mathcal{D}$, under Assumptions 1 and 2, if a Social Ordering Function satisfies Strong Pareto, Nested Preferences Transfer, Equal Preferences Permutation, Equal Talent Transfer to the Unhappy and Separation then the optimal incentive compatible allocation $\left(m_{N}^{t^{H}}\right)$ distributes the available resources in the following way:

$$
\text { for all } i, j \in N,\left(m_{i}^{t^{H}}, t_{i}\right) I^{H}\left(m_{j}^{t^{H}}, t_{j}\right) \text { or } m_{i}=0 \text { and }\left(0, t_{i}\right) R^{H}\left(m_{j}, t_{j}\right)
$$

Such a compensation path gives maximal priority to agents with a lower talent leaving a relatively big share of the population without any compensation (see figure $5(\mathrm{c})$ ).

\section{Conclusions}

In the first part of this paper we have examined how axioms embodying the principle of compensation and axioms weakly embodying the principle of natural reward lead to a particular way of evaluating individual welfare and single out particular social preferences. Such social preferences grant absolute priority to agents who dislike their level of talent and to agents who receive a low compensation. The measure of individual situations obtained here is derived from the fairness principles and the analysis did not require any other information about individual welfare than ordinal non-comparable preferences.

It must be stressed again that alternative measures of individual welfare and alternative social preferences, both responding to different ethical principles, could be found. The purpose of our analysis was to see how one could derive specific policies recommendations from given fairness principles.

The second part of this paper has studied the implications of such social preferences for the evaluation of incentive compatible-policies. The fact that incentive constraints prevent the full expression of priority given to the principle of compensation does not render the principle irrelevant. On the contrary it tells us to what extent an implementable policy can take this ethical requirement into account.

The main lesson we can draw from this paper is in fact that leaving a certain share of the population without a monetary compensation can be defended on normative grounds, given the scarcity of the available resources 
and the informational constraints. In particular this share is endogenously determined if we chose a particular weakening of Equal Talent Transfer.

Finally let us briefly discuss the assumptions underlying the analysis. In Fleurbaey [7] [8] no particular structure is assumed on the set $T$. Here Lemma 1,2 and 3 would still hold under the same assumption too. In our analysis assuming that $T$ has at least some ordinal structure plays a fundamental role in the way the social ordering function we propose is built. As we have seen, many compensation schemes used in reality are based on indexes that induce a structure on individual talents and handicaps.

\section{Appendix: proofs}

Proof of Lemma 1. Take the economy $e=\left(t, t^{\prime}, t^{\prime}, t, R^{\prime}, R^{\prime}, R, R, M\right) \in$ $\mathcal{D}$, the extended bundles $\left(m^{1}, t\right),\left(m^{2}, t\right),\left(m^{3}, t\right),\left(m^{4}, t\right),\left(m^{5}, t^{\prime}\right),\left(m^{6}, t^{\prime}\right)$, $\left(m^{7}, t^{\prime}\right),\left(m^{8}, t^{\prime}\right)$ and $\Delta \in \mathbb{R}_{++}$with:

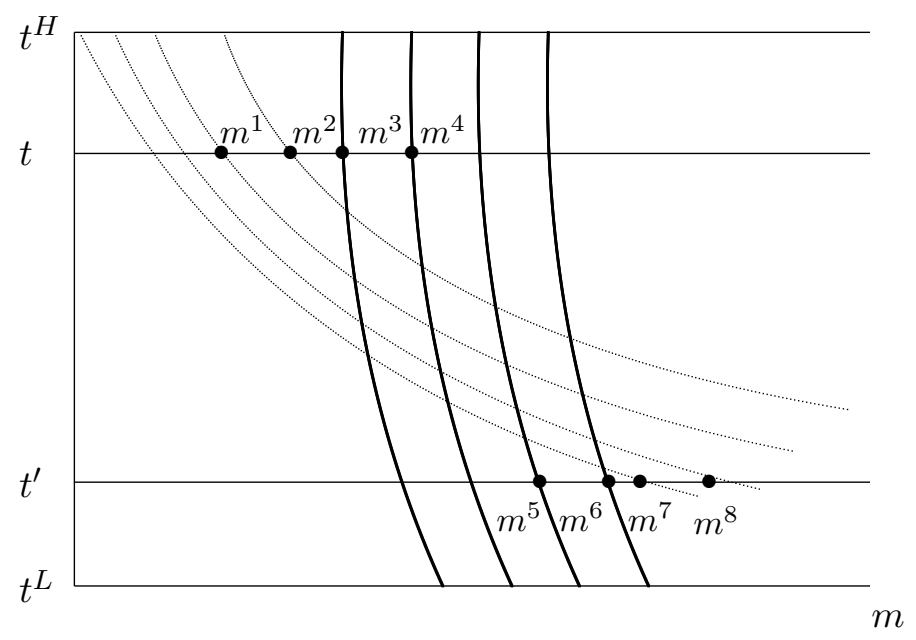

Figure 6: Equal Preferences Transfer and Equal Talent Transfer are incompatible.

1. $t \neq t^{\prime}$

2. $\left(m^{1}, t\right) P\left(m^{8}, t^{\prime}\right)$

3. $\left(m^{5}, t^{\prime}\right) P^{\prime}\left(m^{4}, t\right)$

4. $m^{4}-\Delta=m^{3}>m^{2}=m^{1}+\Delta$ 
5. $m^{8}-\Delta=m^{7}>m^{6}=m^{5}+\Delta$

We give a graphical example of such an economy in figure 6. By Equal Preferences Transfer and conditions 2, 4 and $5,\left(m^{1}, m^{8}, m^{5}, m^{3}\right) \bar{P}(e)\left(m^{2}, m^{7}\right.$, $\left.m^{5}, m^{3}\right)$. By Equal Talent Transfer and condition $5,\left(m^{1}, m^{7}, m^{6}, m^{3}\right) \bar{P}(e)$ $\left(m^{1}, m^{8}, m^{5}, m^{3}\right)$. By Equal Preferences Transfer and conditions 3, 4 and $5,\left(m^{1}, m^{7}, m^{5}, m^{4}\right) \bar{P}(e)\left(m^{1}, m^{7}, m^{6}, m^{3}\right)$. By Equal Talent Transfer and condition $4,\left(m^{2}, m^{7}, m^{5}, m^{3}\right) \bar{P}(e)\left(m^{1}, m^{7}, m^{5}, m^{4}\right)$. Finally, by transitivity, $\left(m^{1}, m^{7}, m^{6}, m^{3}\right) \bar{P}(e)\left(m^{1}, m^{7}, m^{6}, m^{3}\right)$, which yields the desired contradiction.

Proof of Lemma 2. Take the economy $e=\left(t, t, t^{\prime}, t^{\prime}, R, R^{\prime}, R^{\prime}, R, M\right) \in \mathcal{D}$, the extended bundles $\left(m^{1}, t\right),\left(m^{2}, t\right),\left(m^{3}, t\right),\left(m^{4}, t\right),\left(m^{5}, t\right),\left(m^{6}, t\right),\left(m^{1^{\prime}}\right.$ ,$\left.t^{\prime}\right),\left(m^{2^{\prime}}, t^{\prime}\right),\left(m^{3^{\prime}}, t^{\prime}\right),\left(m^{4^{\prime}}, t^{\prime}\right),\left(m^{5^{\prime}}, t^{\prime}\right),\left(m^{6^{\prime}}, t^{\prime}\right)$ with:

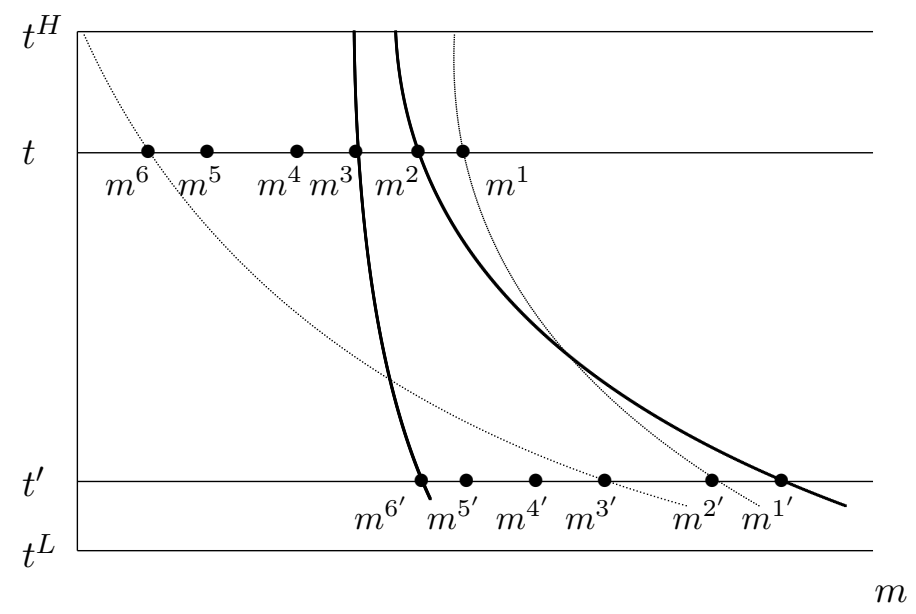

Figure 7: Strong Pareto, Equal Preferences Permutation and Equal Talent Permutation are incompatible.

1. $t \neq t^{\prime}$

2. $m^{1}>m^{2}>m^{3}>m^{4}>m^{5}>m^{6}$

3. $m^{1^{\prime}}>m^{2^{\prime}}>m^{3^{\prime}}>m^{4^{\prime}}>m^{5^{\prime}}>m^{6^{\prime}}$

4. $\left(m^{1}, t\right) I\left(m^{2^{\prime}}, t^{\prime}\right)$ and $\left(m^{6}, t\right) I\left(m^{3^{\prime}}, t^{\prime}\right)$

5. $\left(m^{2}, t\right) I^{\prime}\left(m^{1^{\prime}}, t^{\prime}\right)$ and $\left(m^{3}, t\right) I^{\prime}\left(m^{6^{\prime}}, t^{\prime}\right)$ 
A graphical example of such an economy is given in figure 7. By Strong Pareto and conditions 2 and $3,\left(m^{1}, m^{4}, m^{3^{\prime}}, m^{5^{\prime}}\right) \bar{P}(e)\left(m^{2}, m^{5}, m^{4^{\prime}}, m^{6^{\prime}}\right)$. By Equal Talent Permutation and condition 3, $\left(m^{4}, m^{1}, m^{3^{\prime}}, m^{5^{\prime}}\right) \bar{I}(e)\left(m^{1}\right.$, $\left.m^{4}, m^{3^{\prime}}, m^{5^{\prime}}\right)$. By Equal Preferences Permutation and condition $4,\left(m^{4}, m^{6}\right.$, $\left.m^{2^{\prime}}, m^{5^{\prime}}\right) \bar{I}(e)\left(m^{4}, m^{1}, m^{3^{\prime}}, m^{5^{\prime}}\right)$. By Equal Talent Permutation and condition 3, $\left(m^{4}, m^{6}, m^{2^{\prime}}, m^{5^{\prime}}\right) \bar{I}(e)\left(m^{4}, m^{6}, m^{5^{\prime}}, m^{2^{\prime}}\right)$. By Strong Pareto and conditions 2 and $3,\left(m^{3}, m^{5}, m^{4^{\prime}}, m^{1^{\prime}}\right) \bar{P}(e)\left(m^{4}, m^{6}, m^{5^{\prime}}, m^{2^{\prime}}\right)$. By Equal Preferences Permutation and condition 5, $\left(m^{2}, m^{5}, m^{4^{\prime}}, m^{6^{\prime}}\right) \bar{I}(e)\left(m^{3}, m^{5}\right.$, $\left.m^{4^{\prime}}, m^{1^{\prime}}\right)$. Finally by transitivity, we have $\left(m^{2}, m^{5}, m^{4^{\prime}}, m^{6^{\prime}}\right) \bar{P}(e)$ $\left(m^{2}, m^{5}, m^{4^{\prime}}, m^{6^{\prime}}\right)$, a contradiction.

Proof of Lemma 3. Let $\bar{R}$ satisfy Nested Preferences Transfer, Equal Preferences Permutation and Separation. Let $e=\left(t_{N}, R_{N}, M\right) \in \mathcal{D}, m_{N}, m_{N}^{\prime} \in$ $\mathbb{R}_{+}^{N}, j, k \in N$ be such that:

$$
\begin{gathered}
\left(m_{j}, t_{j}\right) P_{j}\left(m_{j}^{\prime}, t_{j}\right) P_{j}\left(m_{k}^{\prime}, t_{k}\right) P_{k}\left(m_{k}, t_{k}\right), \\
U\left(m_{j}^{\prime}, R_{j}\right) \bigcap L\left(m_{k}^{\prime}, R_{k}\right)=\emptyset
\end{gathered}
$$

and for all $i \neq j, k, m_{i}=m_{i}^{\prime}$. We want to prove that $m_{N}^{\prime} \bar{P}(e) m_{N}$. Let $\triangle_{j}=\left(m_{j}-m_{j}^{\prime}\right)$ and $\triangle_{k}=\left(m_{k}^{\prime}-m_{k}\right)$. Let $b, c \in \mathbb{N}_{++} \backslash N, R_{b}, R_{c} \in$ $\mathcal{R}, t_{b}, t_{c}, m_{b}^{1}, m_{b}^{2}, m_{c}^{1}, m_{c}^{2} \in \mathbb{R}_{+}$and $q \in \mathbb{N}_{++}$, be defined in such a way that: $R_{b}=R_{c} ;\left(m_{b}^{1}, t_{b}\right) I_{b}\left(m_{c}^{1}, t_{c}\right)$ and $\left(m_{b}^{2}, t_{b}\right) I_{b}\left(m_{c}^{2}, t_{c}\right) ; m_{b}^{1}=m_{b}^{2}+\frac{\triangle_{k}}{q}$ and $m_{c}^{1}=m_{c}^{2}+\frac{\triangle_{j}}{q} ;\left(m_{b}^{2}, t_{b}\right) P_{b}\left(m_{k}^{\prime}, t_{k}\right), U\left(\left(m_{b}^{2}, t_{b}\right), R_{b}\right) \cap L\left(\left(m_{k}^{\prime}, t_{k}\right), R_{k}\right)=$ $\emptyset, \quad\left(m_{j}^{\prime}, t_{j}\right) P_{j}\left(m_{b}^{1}, t_{b}\right)$ and $U\left(\left(m_{j}^{\prime}, t_{j}\right), R_{j}\right) \cap L\left(\left(m_{b}^{1}, t_{b}\right), R_{b}\right)=\emptyset$. Let $e^{\prime}=$ $\left(t_{N}, t_{b}, t_{c}, R_{N}, R_{b}, R_{c}, M\right) \in \mathcal{D}$ (an example of such a construction is given in figure 8).

By Nested Preferences Transfer,

$$
\left(m_{N \backslash\{k\}}, m_{k}+\frac{\triangle_{k}}{q}, m_{b}^{2}, m_{c}^{1}\right) \bar{P}\left(e^{\prime}\right)\left(m_{N}, m_{b}^{1}, m_{c}^{1}\right) .
$$

By Equal Preferences Permutation,

$$
\left(m_{N \backslash\{k\}}, m_{k}+\frac{\triangle_{k}}{q}, m_{b}^{1}, m_{c}^{2}\right) \bar{I}\left(e^{\prime}\right)\left(m_{N \backslash\{k\}}, m_{k}+\frac{\triangle_{k}}{q}, m_{b}^{2}, m_{c}^{1}\right) .
$$

By Nested Preferences Transfer,

$$
\left(m_{N \backslash\{k, j\}}, m_{k}+\frac{\triangle_{k}}{q}, m_{j}-\frac{\triangle_{j}}{q}, m_{b}^{1}, m_{c}^{1}\right) \bar{P}\left(e^{\prime}\right)\left(m_{N \backslash\{k\}}, m_{k}+\frac{\triangle_{k}}{q}, m_{b}^{1}, m_{c}^{2}\right) .
$$

By transitivity,

$$
\left(m_{N \backslash\{k, j\}}, m_{k}+\frac{\triangle_{k}}{q}, m_{j}-\frac{\triangle_{j}}{q}, m_{b}^{1}, m_{c}^{1}\right) \bar{P}\left(e^{\prime}\right)\left(m_{N}, m_{b}^{1}, m_{c}^{1}\right) .
$$




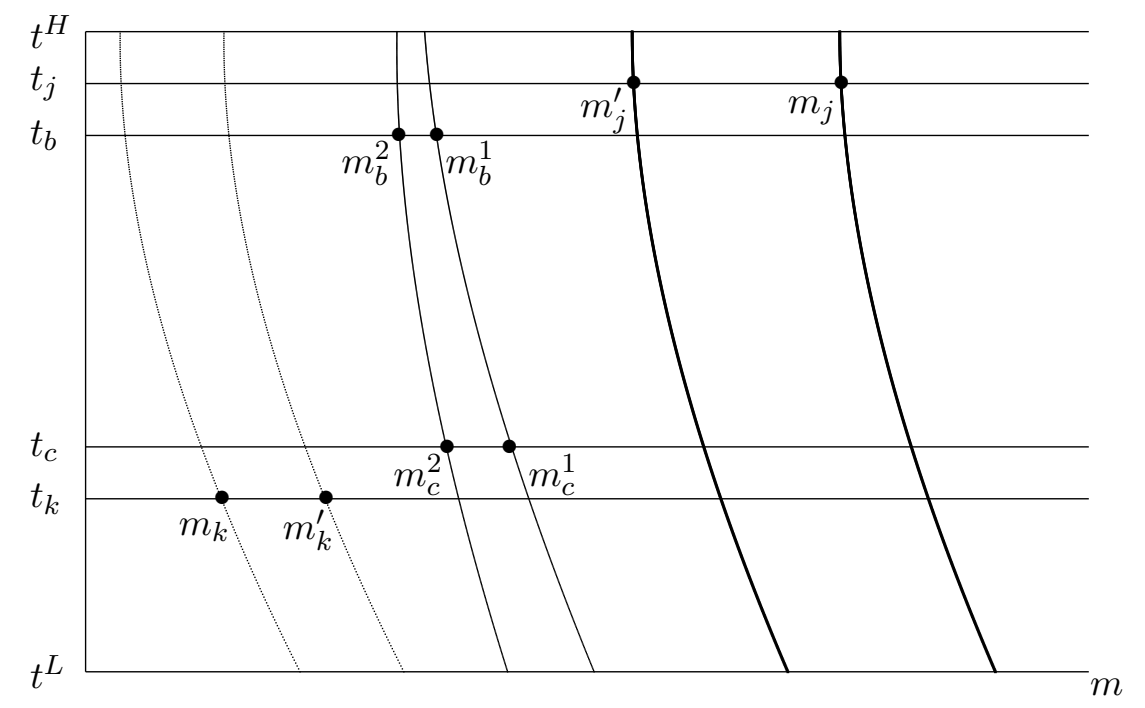

Figure 8: From Nested Preferences Transfer to Nested Preferences Priority.

Replicating the argument $q$ times yields

$$
\left(m_{N \backslash\{k, j\}}, m_{k}+\triangle_{k}, m_{j}-\triangle_{j}, m_{b}^{1}, m_{c}^{1}\right) \bar{P}\left(e^{\prime}\right)\left(m_{N}, m_{b}^{1}, m_{c}^{1}\right) .
$$

Since $m_{j}-\triangle_{j}=m_{j}^{\prime}$ and $m_{k}+\triangle_{k}=m_{k}^{\prime}$, by replacing into the former equation one obtains

$$
\left(m_{N}^{\prime}, m_{b}^{1}, m_{c}^{1}\right) \bar{P}\left(e^{\prime}\right)\left(m_{N}, m_{b}^{1}, m_{c}^{1}\right) .
$$

Finally, by Separation,

$$
m_{N}^{\prime} \bar{P}(e) m_{N}
$$

the desired result.

Proof of Theorem 1. Let $\bar{R}$ be a social ordering function that satisfies Strong Pareto, Nested Preferences Transfer, Equal Preferences Permutation, $\widetilde{t}$-Equal Talent Transfer and Separation. Choose any $\widetilde{t} \in \mathbb{R}_{+}$. By Lemma 3 , the social ordering function $\bar{R}$ also satisfies Nested Preferences Priority. For the sake of clarity the remaining of the proof is divided in two steps.

Step 1. Consider two allocations $m_{N}$ and $m_{N}^{\prime}$ and two different agents $j$ and $k$ such that for all $i \neq j, k, m_{i}=m_{i}^{\prime}$. Let

$$
\widehat{E}_{m}=\min \left\{\widehat{E}\left(m_{j}, t_{j}, R_{j}, \widetilde{t}\right), \widehat{E}\left(m_{j}^{\prime}, t_{j}, R_{j}, \widetilde{t}\right), \widehat{E}\left(m_{k}^{\prime}, t_{k}, R_{k}, \widetilde{t}\right) \cdot\right\}
$$


Without loss of generality, let us assume that $\widehat{E}\left(m_{k}, t_{k}, R_{k}, \widetilde{t}\right)<\widehat{E}_{m}$. We want to prove that $m_{N}^{\prime} \bar{P}(e) m_{N}$. If we also have $m_{j}^{\prime} \geqslant m_{j}$ then by Strong Pareto we immediately get the desired result. Consider now the case $m_{j}>$ $m_{j}^{\prime}$ and assume, by contradiction, that $m_{N} \bar{R}(e) m_{N}^{\prime}$. We have to examine two possible cases.

- Case 1: $\widehat{E}\left(m_{k}, t_{k}, R_{k}, \widetilde{t}\right)<0$.

Let $b, c \in \mathbb{N}_{++} \backslash N, R_{b}, R_{c} \in \mathcal{R}, t_{b}, t_{c} \in T, m_{b}, m_{c}, m_{k}^{\prime \prime} \in \mathbb{R}_{+}$be defined in such a way that: (a graphical example of the following construction is given in figure 9):

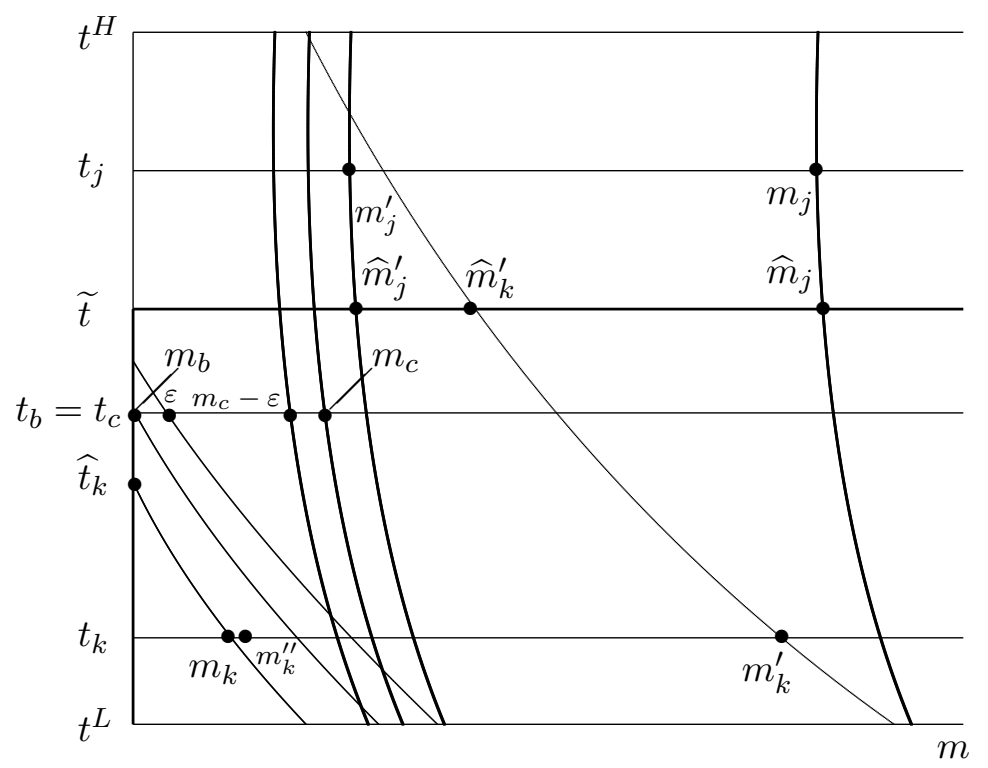

Figure 9: Case 1.

1. $\widehat{t}_{k}<t_{b}=t_{c}<\tilde{t}$ if $\widehat{m}_{m}$ exists, $\widehat{t}_{k}<t_{b}=t_{c}<\widehat{t}_{m}$ otherwise

2. $R_{k}=R_{b}$ and $R_{j}=R_{c}$

3. $0=m_{b}<m_{c}$ and $\left(m_{j}^{\prime}, t_{j}\right) P_{j}\left(m_{c}, t_{c}\right)$

4. $m_{k}<m_{k}^{\prime \prime}<m_{k}^{\prime}$ and $\left(m_{b}, t_{b}\right) P_{k}\left(m_{k}^{\prime \prime}, t_{k}\right)$.

Let $e^{\prime}=\left(t_{j}, t_{k}, R_{j}, R_{k}, M\right), e^{\prime \prime}=\left(t_{j}, t_{k}, t_{b}, t_{c}, R_{j}, R_{k}, R_{b}, R_{c}, M\right) \in \mathcal{D}$. Since we have assumed $m_{N} \bar{R}(e) m_{N}^{\prime}$ then, by Separation, we have $\left(m_{j}, m_{k}\right)$ $\bar{R}\left(e^{\prime}\right)\left(m_{j}^{\prime}, m_{k}^{\prime}\right)$. Take any $\varepsilon$ such that $0<\varepsilon<\frac{m_{c}}{2}$. By Separation again $\left(m_{j}, m_{k}, m_{b}+\varepsilon, m_{c}-\varepsilon\right) \bar{R}\left(e^{\prime \prime}\right)\left(m_{j}^{\prime}, m_{k}^{\prime}, m_{b}+\varepsilon, m_{c}-\varepsilon\right)$. By Nested Preferences 
Priority and conditions 2 and $3,\left(m_{j}^{\prime}, m_{k}, m_{b}+\varepsilon, m_{c}\right) \bar{P}\left(e^{\prime \prime}\right)\left(m_{j}, m_{k}, m_{b}+\right.$ $\left.\varepsilon, m_{c}-\varepsilon\right)$. By Nested Preferences Priority and conditions 2 and $4,\left(m_{j}^{\prime}, m_{k}^{\prime \prime}\right.$, $\left.m_{b}, m_{c}\right) \bar{P}\left(e^{\prime \prime}\right) \quad\left(m_{j}^{\prime}, m_{k}, m_{b}+\varepsilon, m_{c}\right)$. By Strong Pareto and condition 4, $\left(m_{j}^{\prime}, m_{k}^{\prime}, m_{b}, m_{c}\right) \bar{P}\left(e^{\prime \prime}\right)\left(m_{j}^{\prime}, m_{k}^{\prime \prime}, m_{b}, m_{c}\right)$. By Transitivity, $\left(m_{j}^{\prime}, m_{k}^{\prime}, m_{b}, m_{c}\right)$ $\bar{P}\left(e^{\prime \prime}\right)\left(m_{j}^{\prime}, m_{k}^{\prime}, m_{b}+\varepsilon, m_{c}-\varepsilon\right)$. This is true for each $\varepsilon$ such that $0<\varepsilon<\frac{m_{c}}{2}$ hence, given condition 1 and 3 , it represents a violation of $\widetilde{t}$-Equal Talent Transfer and yields the desired contradiction so that $m_{N}^{\prime} \bar{P}(e) m_{N}$

- Case 2: $\widehat{E}\left(m_{k}, t_{k}, R_{k}, \widetilde{t}\right) \geq 0$.

Let $b, c \in \mathbb{N}_{++} \backslash N, R_{b}, R_{c} \in \mathcal{R}, t_{b}, t_{c} \in T, m_{b}, m_{b}^{\prime}, m_{c}, m_{c}^{\prime}, m_{k}^{\prime \prime} \in \mathbb{R}_{+}, \Delta \in$ $\mathbb{R}_{++}$be defined in such a way that: (for a graphical example of the following construction see figure 10):

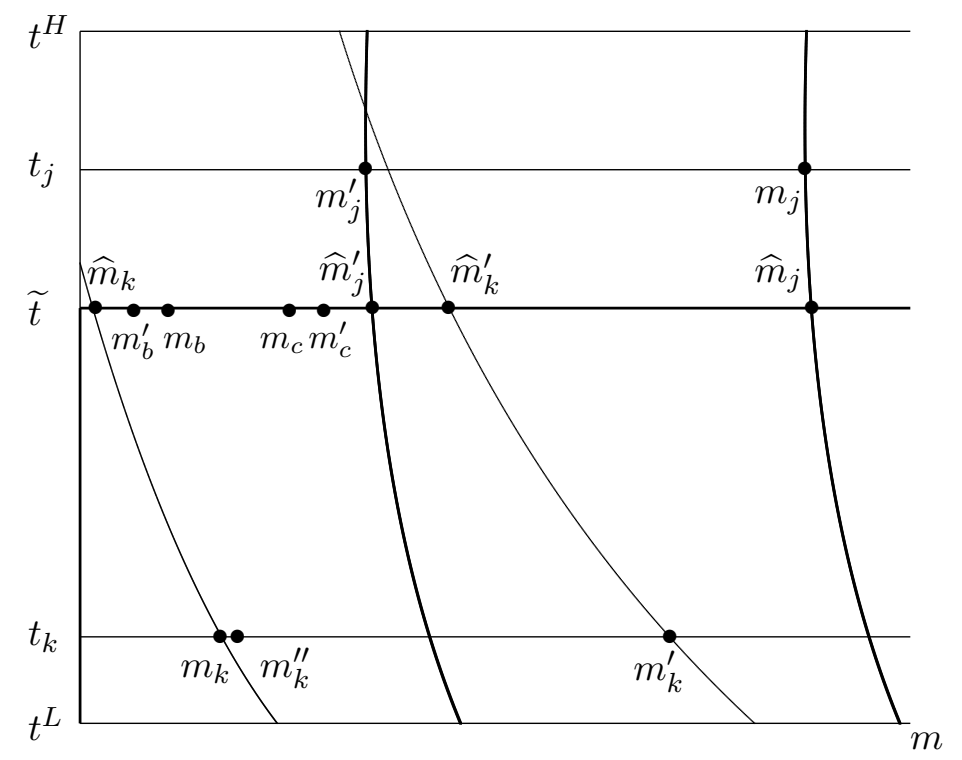

Figure 10: Case 2.

(5) $t_{b}=t_{c}=\tilde{t}$

(6) $R_{k}=R_{b}$ and $R_{j}=R_{c}$

(7) $\widehat{E}\left(m_{k}, t_{k}, R_{k}, \widetilde{t}\right)<m_{b}^{\prime}<m_{c}^{\prime}<\widehat{E}_{m}$ and $m_{b}^{\prime}+\Delta=m_{b}<m_{c}=m_{c}^{\prime}-\Delta$

(8) $m_{k}<m_{k}^{\prime \prime}$ and $\left(m_{b}, t_{b}\right) P_{k}\left(m_{k}^{\prime \prime}, t_{k}\right)$. 
Let $e^{\prime}=\left(t_{j}, t_{k}, R_{j}, R_{k}, M\right), e^{\prime \prime}=\left(t_{j}, t_{k}, t_{b}, t_{c}, R_{j}, R_{k}, R_{b}, R_{c}, M\right) \in \mathcal{D}$. Since we have assumed $m_{N} \bar{R}(e) m_{N}^{\prime}$ then, by Separation we have respectively, $\left(m_{j}, m_{k}\right) \bar{R}\left(e^{\prime}\right)\left(m_{j}^{\prime}, m_{k}^{\prime}\right)$ and $\left(m_{j}, m_{k}, m_{b}, m_{c}\right) \bar{R}\left(e^{\prime \prime}\right)\left(m_{j}^{\prime}, m_{k}^{\prime}\right.$, $\left.m_{b}, m_{c}\right)$. By Nested Preferences Priority and conditions 6 and $7,\left(m_{j}^{\prime}, m_{k}\right.$, $\left.m_{b}, m_{c}^{\prime}\right) \bar{P}\left(e^{\prime \prime}\right)\left(m_{j}, m_{k}, m_{b}, m_{c}\right)$. By Nested Preferences Priority and conditions 6,7 and 8, $\left(m_{j}^{\prime}, m_{k}^{\prime \prime}, m_{b}^{\prime}, m_{c}^{\prime}\right) \bar{P}\left(e^{\prime \prime}\right)\left(m_{j}^{\prime}, m_{k}, m_{b}, m_{c}^{\prime}\right)$. By Strong Pareto and condition $8,\left(m_{j}^{\prime}, m_{k}^{\prime}, m_{b}^{\prime}, m_{c}^{\prime}\right) \bar{P}\left(e^{\prime \prime}\right)\left(m_{j}^{\prime}, m_{k}^{\prime \prime}, m_{b}^{\prime}, m_{c}^{\prime}\right)$. By Transitivity, $\left(m_{j}^{\prime}, m_{k}^{\prime}, m_{b}^{\prime}, m_{c}^{\prime}\right) \bar{P}\left(e^{\prime \prime}\right)\left(m_{j}^{\prime}, m_{k}^{\prime}, m_{b}, m_{c}\right)$, which, given conditions 5 and 7 , is a violation of $\widetilde{t}$-Equal Talent Transfer and again yields the desired contradiction so that $m_{N}^{\prime} \bar{P}(e) m_{N}$.

Step 2. Take now two allocations $m_{N}$ and $m_{N}^{\prime}$ such that

$$
\min _{i} \widehat{E}\left(m_{i}^{\prime}, t_{i}, R_{i}, \widetilde{t}\right)>\min _{i} \widehat{E}\left(m_{i}, t_{i}, R_{i}, \widetilde{t}\right) .
$$

Then, by monotonicity of preferences, one can find two allocations $\bar{m}_{N}, \bar{m}_{N}^{\prime}$ such that for all $i, m_{i}<\bar{m}_{i}, \bar{m}_{i}^{\prime}<m_{i}^{\prime}$, and there exists $i_{0}$ such that for all $i \neq i_{0}$

$$
\widehat{E}\left(\bar{m}_{i}, t_{i}, R_{i}, \widetilde{t}\right)>\widehat{E}\left(\bar{m}_{i}^{\prime}, t_{i}, R_{i}, \widetilde{t}\right)>\widehat{E}\left(\bar{m}_{i_{0}}^{\prime}, t_{i_{0}}, R_{i_{0}}, \widetilde{t}\right)>\widehat{E}\left(\bar{m}_{i_{0}}, t_{i_{0}}, R_{i_{0}}, \widetilde{t}\right) .
$$

Let $Q=N \backslash\left\{i_{0}\right\}$ and consider a sequence of allocations $\left(\bar{m}_{N}^{q}\right)_{1 \leq q \leq|Q|+1}$ such that

$$
\begin{aligned}
& \bar{m}_{i}^{q}=\bar{m}_{i}^{\prime} \text { for all } i \in Q \text { such that } i<q \\
& \bar{m}_{i}^{q}=\bar{m}_{i} \text { for all } i \in Q \text { such that } i \geq q
\end{aligned}
$$

while

$$
\bar{m}_{i_{0}}^{\prime}=\bar{m}_{i_{0}}^{|Q|+1}>\bar{m}_{i_{0}}^{|Q|}>\ldots>\bar{m}_{i_{0}}^{1}=\bar{m}_{i_{0}} .
$$

This entails that for all $q \in Q$

$$
\begin{gathered}
\widehat{E}\left(\bar{m}_{q}^{q}, t_{m}, R_{m}, \widetilde{t}\right)>\widehat{E}\left(\bar{m}_{q}^{q+1}, t_{m}, R_{k}, \widetilde{t}\right)>\widehat{E}\left(\bar{m}_{i_{0}}^{q+1}, t_{i_{0}}, R_{i_{0}}, \widetilde{t}\right)> \\
\widehat{E}\left(\bar{m}_{i_{0}}^{q}, t_{i_{0}}, R_{i_{0}}, \widetilde{t}\right),
\end{gathered}
$$

while for all $q \in Q$, and all $i \neq i_{0}, q, \bar{m}_{i}^{q}=\bar{m}_{i}^{q+1}$. By Step $1, \bar{m}_{N}^{q+1} P(e) \bar{m}_{N}^{q}$ for all $q \in Q$. By Strong Pareto, $m_{N}^{\prime} P(e) \bar{m}_{N}^{|Q|+1}$ and $\bar{m}_{N}^{1} P(e) m_{N}$. By transitivity, $m_{N}^{\prime} P(e) m_{N}$.

Examples of social ordering functions satisfying all the axioms but:

1. Strong Pareto. Compare the welfare-level vectors using the lexicographic minimax criterion: a distribution is at least as good as another one if its maximum is lower, or they are equal and the second highest value is lower, or... or they are all equal. 
2. Nested Preferences Transfer. Let $\bar{R}$ be defined by: for all $e \in \mathcal{D}$, $m_{N}, m_{N}^{\prime} \in \mathbb{R}_{N}^{+}$, if

$$
\left(\widehat{E}\left(m_{i}^{\prime}, t_{i}, R_{i}, \widetilde{t}\right)\right)_{i \in N}>_{L}\left(\widehat{E}\left(m_{i}, t_{i}, R_{i}, \widetilde{t}\right)\right)_{i \in N}
$$

and there exist $k, k^{\prime} \in N$ with $t_{k} \neq t_{k^{\prime}}$ such that

$$
\begin{gathered}
0<\widehat{E}\left(m_{k}, t_{k}, R_{k}, \widetilde{t}\right)<\widehat{E}\left(m_{k}^{\prime}, t_{i}, R_{i}, \widetilde{t}\right)<\widehat{E}\left(m_{k^{\prime}}^{\prime}, t_{k}, R_{k}, \widetilde{t}\right)< \\
\widehat{E}\left(m_{k^{\prime}}, t_{i}, R_{i}, \widetilde{t}\right),
\end{gathered}
$$

with $\widehat{E}\left(m_{i}^{\prime}, t_{i}, R_{i}, \widetilde{t}\right)=\widehat{E}\left(m_{i}, t_{i}, R_{i}, \widetilde{t}\right)$ for all $i \neq k, k^{\prime}$, then $m_{N}^{\prime} \bar{I}(e) m_{N}$. In all other cases $m_{N}^{\prime} \bar{P}(e) m_{N}$. If

$$
\left(\widehat{E}\left(m_{i}^{\prime}, t_{i}, R_{i}, \widetilde{t}\right)\right)_{i \in N}={ }_{L}\left(\widehat{E}\left(m_{i}, t_{i}, R_{i}, \widetilde{t}\right)\right)_{i \in N}
$$

then $m_{N}^{\prime} \bar{I}(e) m_{N}$.

3. Equal Preferences Permutation. Let $\bar{R}$ be defined by: for all $e \in \mathcal{D}$, $m_{N}, m_{N}^{\prime} \in \mathbb{R}_{N}^{+}$, if

$$
\left(\widehat{E}\left(m_{i}^{\prime}, t_{i}, R_{i}, \widetilde{t}\right)\right)_{i \in N}>_{L}\left(\widehat{E}\left(m_{i}, t_{i}, R_{i}, \widetilde{t}\right)\right)_{i \in N}
$$

or

$$
\left(\widehat{E}\left(m_{i}^{\prime}, t_{i}, R_{i}, \widetilde{t}\right)\right)_{i \in N}={ }_{L}\left(\widehat{E}\left(m_{i}, t_{i}, R_{i}, \widetilde{t}\right)\right)_{i \in N}
$$

and there exist $k, k^{\prime} \in N$ with $t_{k^{\prime}} \neq t_{k}$ such that $\widehat{E}\left(m_{k}, t_{k}, R_{k}, \widetilde{t}\right)<$ $\widehat{E}\left(m_{i}, t_{i}, R_{i}, \widetilde{t}\right)$ for all $i \neq k ; \widehat{E}\left(m_{k^{\prime}}^{\prime}, t_{k^{\prime}}, R_{k^{\prime}}, \widetilde{t}\right)<\widehat{E}\left(m_{i}^{\prime}, t_{i}, R_{i}, \widetilde{t}\right)$ for all $i \neq k^{\prime}$, then $m_{N}^{\prime} \bar{P}(e) m_{N}$. In all other cases $m_{N}^{\prime} \bar{I}(e) m_{N}$.

4. $\widetilde{t}$-Equal Talent Transfer. Let $\bar{R}$ be defined by: for all $e \in \mathcal{D}, m_{N}, m_{N}^{\prime} \in$ $\mathbb{R}_{N}^{+}$, if

$$
\left(\widehat{E}\left(m_{i}^{\prime}, t_{i}, R_{i}, \widetilde{t}\right)\right)_{i \in N}>_{L}\left(\widehat{E}\left(m_{i}, t_{i}, R_{i}, \widetilde{t}\right)\right)_{i \in N}
$$

and there exist $k, k^{\prime} \in N$ with $R_{k^{\prime}} \neq R_{k}$ such that

$$
\begin{gathered}
\widehat{E}\left(m_{k}, t_{k}, R_{k}, \widetilde{t}\right)<\widehat{E}\left(m_{k}^{\prime}, t_{i}, R_{i}, \widetilde{t}\right)<\widehat{E}\left(m_{k^{\prime}}^{\prime}, t_{k}, R_{k}, \widetilde{t}\right)< \\
\widehat{E}\left(m_{k^{\prime}}, t_{i}, R_{i}, \widetilde{t}\right),
\end{gathered}
$$

with $\widehat{E}\left(m_{i}^{\prime}, t_{i}, R_{i}, \widetilde{t}\right)=\widehat{E}\left(m_{i}, t_{i}, R_{i}, \widetilde{t}\right)$ for all $i \neq k, k^{\prime}$ and $U\left(\left(m_{k^{\prime}}^{\prime}, t_{k}^{\prime}\right), R_{k}^{\prime}\right)$ $\bigcap L\left(\left(m_{k}^{\prime}, t_{k}\right), R_{k}\right) \neq \emptyset$, then $m_{N}^{\prime} \bar{I}(e) m_{N}$. In all other cases $m_{N}^{\prime} \bar{P}(e) m_{N}$. If

$$
\left(\widehat{E}\left(m_{i}^{\prime}, t_{i}, R_{i}, \widetilde{t}\right)\right)_{i \in N}={ }_{L}\left(\widehat{E}\left(m_{i}^{\prime}, t_{i}, R_{i}, \widetilde{t}\right)\right)_{i \in N}
$$

then $m_{N}^{\prime} \bar{I}(e) m_{N}$. 
5. Separation. Let $\bar{R}$ be defined by: in all economies such that there is an agent $k \in N$ such that $t_{k}=\widetilde{t}$, for all $m_{N}, m_{N}^{\prime} \in \mathbb{R}_{N}^{+}$

$$
\left(\widehat{E}\left(m_{i}^{\prime}, t_{i}, R_{i}, \widetilde{t}\right)\right)_{i \in N} \geq_{L}\left(\widehat{E}\left(m_{i}, t_{i}, R_{i}, \widetilde{t}\right)\right)_{i \in N} \Longleftrightarrow m_{N}^{\prime} \bar{R}(e) m_{N} .
$$

In all other economies

$$
\left(\widehat{E}\left(m_{i}^{\prime}, t_{i}, R_{i}, \widetilde{t}^{\prime}\right)\right)_{i \in N} \geq_{L}\left(\widehat{E}\left(m_{i}, t_{i}, R_{i}, \widetilde{t}^{\prime}\right)\right)_{i \in N} \Longleftrightarrow m_{N}^{\prime} \bar{R}(e) m_{N}
$$

with $\widetilde{t} \neq \widetilde{t^{\prime}}$.

Proof of Theorem 2. The proof mostly parallels the proof of theorem 1 . We only need to prove case 2 of step 1 which is now divided in two different subcases:

- Case 2a: $\widehat{E}\left(m_{k}, t_{k}, R_{k}, \widetilde{t}\right) \geq 0$ and $R_{k} \succ_{A} R_{j}$.

Let $b, c \in \mathbb{N}_{++} \backslash N, R_{b}, R_{c} \in \mathcal{R}, t_{b}, t_{c} \in T, m_{b}, m_{b}^{\prime}, m_{c}, m_{c}^{\prime}, m_{k}^{\prime \prime} \in \mathbb{R}_{+}, \Delta \in$ $\mathbb{R}_{++}$be defined in such a way that (see figure 11 ):

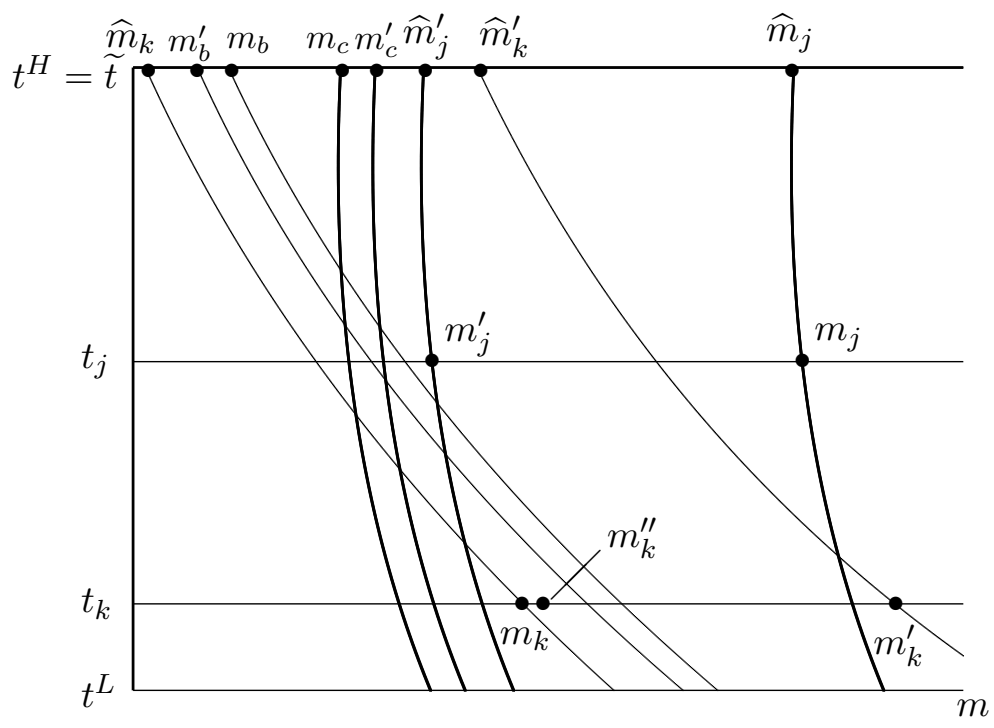

Figure 11: Case 2a.

1. $t_{b}=t_{c}=\widetilde{t}=t^{H}$

2. $R_{k}=R_{b}$ and $R_{j}=R_{c}$

3. $\widehat{E}\left(m_{k}, t_{k}, R_{k}, \widetilde{t}\right)<m_{b}^{\prime}<m_{c}^{\prime}<\widehat{E}_{m}$ and $m_{b}^{\prime}+\Delta=m_{b} \leq m_{c}=m_{c}^{\prime}-\Delta$ 
4. $m_{k}<m_{k}^{\prime \prime}$ and $\left(m_{b}, t_{b}\right) P_{k}\left(m_{k}^{\prime \prime}, t_{k}\right)$.

Let $e^{\prime}=\left(t_{j}, t_{k}, R_{j}, R_{k}, M\right), e^{\prime \prime}=\left(t_{j}, t_{k}, t_{b}, t_{c}, R_{j}, R_{k}, R_{b}, R_{c}, M\right) \in \mathcal{D}$. As in the previous proof we assume, $m_{N} \bar{R}(e) m_{N}^{\prime}$. By applying Separation twice we have $\left(m_{j}, m_{k}\right) \bar{R}\left(e^{\prime}\right)\left(m_{j}^{\prime}, m_{k}^{\prime}\right)$ and $\left(m_{j}, m_{k}, m_{b}, m_{c}\right) \bar{R}\left(e^{\prime \prime}\right)\left(m_{j}^{\prime}, m_{k}^{\prime}, m_{b}\right.$, $\left.m_{c}\right)$. By Nested Preferences Priority and conditions 2, 3 and $4,\left(m_{j}^{\prime}, m_{k}, m_{b}\right.$, $\left.m_{c}^{\prime}\right) \bar{P}\left(e^{\prime \prime}\right)\left(m_{j}, m_{k}, m_{b}, m_{c}\right)$ and $\left(m_{j}^{\prime}, m_{k}^{\prime \prime}, m_{b}^{\prime}, m_{c}^{\prime}\right) \bar{P}\left(e^{\prime \prime}\right) \quad\left(m_{j}^{\prime}, m_{k}, m_{b}, m_{c}^{\prime}\right)$. By Strong Pareto and condition $4,\left(m_{j}^{\prime}, m_{k}^{\prime}, m_{b}^{\prime}, m_{c}^{\prime}\right) \bar{P}\left(e^{\prime \prime}\right)\left(m_{j}^{\prime}, m_{k}^{\prime \prime}, m_{b}^{\prime}, m_{c}^{\prime}\right)$. By Transitivity, $\left(m_{j}^{\prime}, m_{k}^{\prime}, m_{b}^{\prime}, m_{c}^{\prime}\right) \bar{P}\left(e^{\prime \prime}\right)\left(m_{j}^{\prime}, m_{k}^{\prime}, m_{b}, m_{c}\right)$, which, given conditions 1 and 2, is a violation of Equal Talent Transfer to the Unhappy since, by construction, $R_{b} \succ_{A} R_{c}$. This yields the desired contradiction so that $m_{N}^{\prime} \bar{P}(e) m_{N}$.

- Case 2b: $\widehat{E}\left(m_{k}, t_{k}, R_{k}, \widetilde{t}\right) \geq 0$. and $R_{j} \succ_{A} R_{k}$.

If we have $\widehat{E}\left(m_{k}^{\prime}, t_{k}, R_{k}, \widetilde{t}\right)<\widehat{E}\left(m_{j}^{\prime}, t_{j}, R_{j}, \widetilde{t}\right)$ then directly by Nested Preference Transfer we get the desired result. On the other hand assume

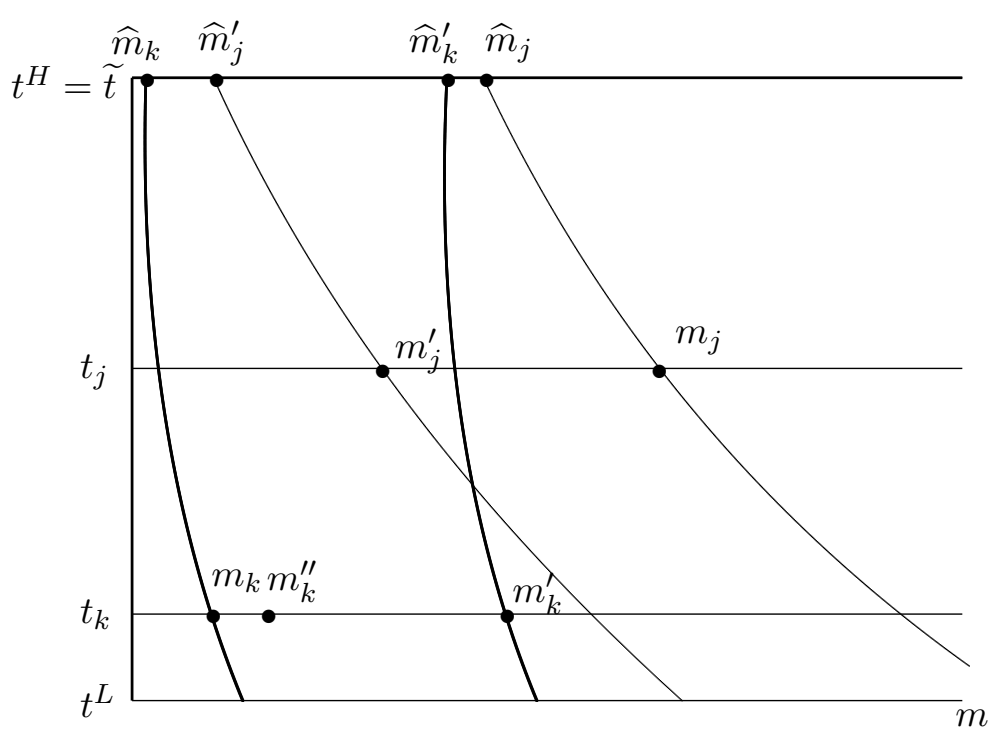

Figure 12: Case 2b.

$\widehat{E}\left(m_{k}^{\prime}, t_{k}, R_{k}, \widetilde{t}\right) \geq \widehat{E}\left(m_{j}^{\prime}, t_{j}, R_{j}, \widetilde{t}\right)$. and, again by contradiction $m_{N} \bar{R}(e) m_{N}^{\prime}$. Consider $m_{k}^{\prime \prime}$ such that $m_{k}<m_{k}^{\prime \prime}<m_{k}^{\prime},\left(m_{j}^{\prime}, t_{j}\right) P_{j}\left(m_{k}^{\prime \prime}, t_{k}\right)$ and $U\left(\left(m_{j}^{\prime}, t_{j}\right), R_{j}\right)$ $\bigcap L\left(\left(m_{k}^{\prime \prime}, t_{k}\right), R_{k}\right)=\emptyset$. Let $e^{\prime}=\left(t_{j}, t_{k}, R_{j}, R_{k}, M\right)$. By Separation we have $\left(m_{j}, m_{k}\right) \bar{R}\left(e^{\prime}\right)\left(m_{j}^{\prime}, m_{k}^{\prime}\right)$. By Nested Preferences Priority, $\left(m_{k}^{\prime \prime}, m_{j}^{\prime}\right) \bar{P}\left(e^{\prime}\right)$ $\left(m_{k}, m_{j}\right)$. By Strong Pareto, $\left(m_{k}^{\prime}, m_{j}^{\prime}\right) P\left(e^{\prime}\right)\left(m_{k}, m_{j}\right)$ which yields the de- 
sired contradiction. The remaining of the proof exactly parallels Step 2 of the previous one.

The examples used previously can be easily adapted here to see that no axiom is redundant.

\section{References}

[1] A.R. Arneson, Equality and equal opportunity for welfare, Philosophical Studies 56(1989) 77-93.

[2] C. d'Aspremont, L.Gevers, Equity and the Informational Basis of Collective Choice, Review of Economic Studies 44(1977) 199-209.

[3] W. Bossert, M. Fleurbaey, D. Van de Gaer, Responsibility, talent, and compensation: a second best analysis, Review of Economic Design 4(1999) 35-56.

[4] G. A. Cohen, On the currency of egalitarian justice, Ethics 99(1989) 906- 944.

[5] S.R. Chakravarty, Ethical Social Index Numbers, Springer, New York, 1990

[6] R. Dworkin, What is Equality? Part 1: Equality of Welfare; Part 2: Equality of Resources, Philosophy and Public Affairs 10(1981) 185-246 and 283-345.

[7] M. Fleurbaey, On Fair compensation, Theory and Decision 36(1994) 277-307.

[8] M. Fleurbaey, Three solutions to the compensation problem, Journal of Economic Theory 65(1995) 505-521.

[9] M. Fleurbaey, The Pazner-Schmeidler social ordering: a defense, Review of Economic Design 9(2005) 145-166.

[10] M. Fleurbaey, F. Maniquet, Utilitarianism versus fairness in welfare economics, in M. Salles and J. A. Weymark eds, Justice, Political Liberalism and Utilitarianism: Themes from Harsanyi and Rawls, Cambridge U. Press, forthcoming.

[11] M. Fleurbaey, F. Maniquet, Compensation and responsibility, in K.J. Arrow, A.K. Sen and K. Suzumura (Eds), Handbook of Social Choice and Welfare V. II, North-Holland, Amsterdam, forthcoming. 
[12] M. Fleurbaey, F. Maniquet, Fair social orderings, Economic Theory, forthcoming.

[13] M. Fleurbaey, F. Maniquet, Fair social orderings when agents have unequal production skills, Social Choice and Welfare 24(2005) 93-127.

[14] M. Fleurbaey, F. Maniquet, Fair income tax, Review of Economic Studies $73(2006) 55-83$.

[15] P.J.Hammond, Equity, Arrow's conditions and Rawls' difference principle, Econometrica(1976) 44 793-804.

[16] F. Maniquet, On the equivalence between welfarism and equality of opportunity, Social Choice and Welfare 23(2004) 127-147.

[17] F. Maniquet, Y. Sprumont, Fair production and allocation of a nonrival good, Econometrica 72(2004) 627-640.

[18] F. Maniquet, Y. Sprumont, Welfare egalitarianism in non-rival environments, Journal of Economic Theory 120(2004) 155-174.

[19] E. Pazner, D. Schmeidler, Egalitarian Equivalent Allocations: A New Concept of Economic Equity, Quarterly Journal of Economics 92(1978) 671-687.

[20] J.Rawls, Theory of Justice, Harvard U. Press, Cambridge, 1971.

[21] J. E. Roemer, Equality of Opportunity, Harvard University Press, Cambridge, Mass. 1998.

[22] P. Suppes, Some Formal Models of Grading Principles, Synthese 16(1966) 284-306.

[23] W. Thomson, Consistent Allocation Rules, mimeo 2004.

[24] P. van Parijs, Real Freedom for All, Oxford University Press, Oxford, 1995. 